\title{
ON A CERTAIN CLASS OF OPERATOR ALGEBRAS
}

\author{
BY \\ JAMES G. GLIMM
}

Introduction. In this paper we study $C^{*}$-algebras which are the uniform closure of strictly ascending sequences of full $n \times n(n<\infty)$ matrix algebras. We call these algebras uniformly hyperfinite. Factors of type $\mathrm{II}_{1}$ which are the weak closure of such a sequence were first studied in [4], where it was proved that all such factors are isomorphic. The algebras we study are not all isomorphic. In $\$ 1$ we classify uniformly hyperfinite algebras according to algebraic type (1.12) and obtain a characterization of these algebras. In $\$ 2$ we identify the pure states and the pure state space of uniformly hyperfinite algebras. The $w^{*}$-closure of the pure states of one of these algebras is the set of all states of the algebra. This is not the first example of a $C^{*}$-algebra whose set of pure states is not closed, cf. [7]. In $\$ 3$ we classify the irreducible representations of uniformly hyperfinite algebras according to unitary equivalence. In $\$ \S 4$ and 5 we study certain representations of uniformly hyperfinite algebras.

The author is pleased to record his gratitude to Professor R. V. Kadison for many helpful suggestions, for simplification of several proofs and for patient supervision of the research in this paper, which is the author's doctoral dissertation at Columbia University.

We assume all algebras have a unit (denoted by $I$ ). A family $\left\{e_{i j}: i, j=1, \cdots, n\right\}$ of operators on a Hilbert space $\mathfrak{S}$ (always complex) is called a family of matrix units if $e_{i j} e_{k m}=0$ for $j \neq k,=e_{i m}$ for $j=k$, if $\sum_{i} e_{i i}$ $=I$ (the identity operator on $\mathfrak{T}$ ), and if $e_{i j}=e_{j \mathfrak{j}}^{*}$. If $\mathfrak{M}$ is the $C^{*}$-algebra generated by these matrix units, we say $\left\{e_{i j}\right\}$ is a family of matrix units for $\mathfrak{M}$. If $X$ is a subset of $\mathfrak{S}$, we denote by $[X]$ the smallest closed linear subspace of $\mathfrak{S}$ containing $X$. If $E$ is a projection on $\mathfrak{F}$, we also denote by $E$ the set $\{x: x \in \mathfrak{F}, x=E x\}$. A state of a $C^{*}$-algebra is a positive linear functional $f$ which satisfies $f(I)=1$. The set of states of a $C^{*}$-algebra is convex and $w^{*}$ compact. The extreme points are called pure states. If $\mathfrak{M}$ is a self-adjoint linear subspace of a $C^{*}$-algebra $\mathfrak{A}$ and if $I \in \mathfrak{M}$, then a state of $\mathfrak{M}$ is also a positive normalized linear functional. The (pure) states of $\mathfrak{M}$ have extensions to (pure) states of $\mathfrak{A}$. If $\tau$ is a state of $\mathfrak{A}$ then there is a representation $\phi_{r}$ of $\mathfrak{A}$ on a Hilbert space $\mathfrak{S}_{\tau}$ and an $x$ in $\mathfrak{S}_{\tau}$ with $\tau=(\cdot x, x) \circ \phi_{\tau}$ and $\mathfrak{S}_{\tau}=\left[\phi_{\tau}(\mathfrak{A}) x\right]$. $\tau$ is pure if and only if $\phi_{r}$ is irreducible (see [6]). If $f(x)$ is an expression depending upon $x$ and perhaps other variables, we use the notation $f(\cdot)$ to designate the function $x \rightarrow f(x)$.

Received by the editors May 6, 1959. 


\section{Algebraic classification.}

Definition 1.1. Let $\mathfrak{A}$ be a $C^{*}$-algebra. $\mathfrak{A}$ is called uniformly hyperfinite $(U H F)$ of type $\left\{p_{i}\right\}$ if there is a sequence of factors $\left\{\mathfrak{M}_{i}: i=1,2, \cdots\right\}$ in $\mathfrak{A}$ with the following properties:

(1) $\mathfrak{M}_{i}$ is of type $I_{p_{i}}$,

(2) $\mathfrak{M}_{i-1} \subset \mathfrak{M}_{i}$,

(3) $p_{i} \rightarrow \infty$ as $i \rightarrow \infty$,

(4) $\mathfrak{A}$ is the closure of $U_{i} \mathfrak{M}_{i}$.

In this case we shall say that $\mathfrak{A}$ is generated by the factors $\mathfrak{M}_{i}$. $\mathfrak{A}$ is called uniformly hyperfinite if there is a sequence $\left\{p_{i}\right\}$ of positive integers such that $\mathfrak{A}$ is uniformly hyperfinite of type $\left\{p_{i}\right\}$.

We observe that if $\left\{p_{i}\right\}$ is a sequence of positive integers then UHF algebras of type $\left\{p_{i}\right\}$ exist if and only if $p_{i} \mid p_{i+1}$ and $p_{i} \rightarrow \infty$ as $i \rightarrow \infty$. For example, suppose $p_{i}=2^{i}$, let $d$ be an infinite cardinal and let $\mathfrak{S}$ be a $d$-dimensional Hilbert space. Let $E_{1}$ and $E_{2}$ be orthogonal $d$-dimensional projections on $\mathfrak{S}$ with $E_{1}+E_{2}=I$ (the identity operator on $\mathfrak{S}$ ), let $V$ be a partial isometry from $E_{1}$ to $E_{2}$. Then the set of complex linear combinations of $E_{1}, E_{2}, V$ and $V^{*}$ is a factor $\mathfrak{M}_{1}$ of type $I_{2}$. We can choose orthogonal $d$-dimensional projections $F_{1}$ and $F_{2}$ in $E_{1}$ with $F_{1}+F_{2}=E_{1}$, and we can choose a partial isometry $W$ from $F_{1}$ to $F_{2}$. The algebra generated by $\mathfrak{M}_{1}, F_{1}, F_{2}, W$ and $W^{*}$ is a factor $\mathfrak{M}_{2}$ of type $I_{4}$. Continuing in this way, we can construct an ascending sequence $\left\{\mathfrak{M}_{i}\right\}$ of factors on $\mathfrak{S}$, with $\mathfrak{M}_{i}$ of type $I_{p_{i}}\left(p_{i}=2^{i}\right)$. The closure of $\bigcup_{i} \mathfrak{M}_{i}$ (in the norm $\|\cdot\|=\sup \{|(\cdot x, y)|: x, y$ are in the unit sphere of $\mathfrak{S}\}$ ) is a UHF algebra of type $\left\{2^{i}\right\}$. With the next result we begin the classification of UHF algebras under ${ }^{*}$-isomorphisms (see 1.12).

Lemma 1.2. If $\mathfrak{A}$ and $\mathfrak{B}$ are both $U H F$ algebras of type $\left\{p_{i}\right\}$ then $\mathfrak{A}$ and $\mathfrak{B}$ are *-isomorphic.

Proof. $\mathfrak{A}$ is the closure of $U_{i} \mathfrak{M}_{i}, \mathfrak{B}$ is the closure of $U_{i} \mathfrak{N}_{i}$, where $\mathfrak{M}_{i}$ and $\mathfrak{N}_{i}$ are factors of type $I_{p_{i}}$. It is easy to construct a ${ }^{*}$-isomorphism $\phi$ from $\bigcup_{i} \mathfrak{M}_{i}$ onto $U_{i} \mathfrak{R}_{i}$. For example see [4, p. 760]. For each positive integer $i$, $\phi \mid \mathfrak{M}_{i}$ is norm preserving, so $\phi$ has an extension to a ${ }^{*}$-isomorphism of $\mathfrak{A}$ onto $\mathfrak{B}$.

Definition 1.3. Let $\left\{p_{i}\right\}$ be a sequence of positive integers such that $p_{i} \mid p_{i+1}$ and $p_{i} \rightarrow \infty$ as $i \rightarrow \infty$. We define a function $f\left(\left\{p_{i}\right\}\right)$ whose domain is the prime numbers. Let $x$ be a prime number, let

$$
f\left(\left\{p_{i}\right\}\right)(x)=\sup \left\{n: \text { there is an } i \text { with } x^{n} \mid p_{i}\right\} .
$$

We will show that UHF algebras of type $\left\{p_{i}\right\}$ and type $\left\{q_{i}\right\}$ are ${ }^{*}$-isomorphic if and only if $f\left(\left\{p_{i}\right\}\right)=f\left(\left\{q_{i}\right\}\right)$, that is if and only if the same prime powers are factors of the terms of the sequences $\left\{p_{i}\right\}$ and $\left\{q_{i}\right\}$.

Definition 1.4. Let $\left\{p_{i}\right\}$ be a sequence of positive integers such that $p_{i} \mid p_{i+1}$ and $p_{i} \rightarrow \infty$ as $i \rightarrow \infty$. Let $\left\{x_{j}: j=1,2, \cdots\right\}$ be an enumeration of the prime numbers. We define 


$$
q_{k}=\prod_{j=1}^{k} x_{j}^{\min \left\{k, f\left(\left\{p_{i}\right\}\right)\left(x_{j}\right)\right\}} \quad k=1,2, \cdots
$$

Lemma 1.5. If $\mathfrak{A}$ is a UHF algebra of type $\left\{p_{i}\right\}$, it is also UHF of type $\left\{q_{i}\right\}$ with $\left\{q_{i}\right\}$ as defined in 1.4 .

Proof. Let $\mathfrak{A}$ be generated by factors $\mathfrak{M}_{i}$ of type $I_{p_{i}}$. We remark that for every positive integer $i$, there is a positive integer $j$ such that $p_{i} \mid q_{j}$ and $q_{i} \mid p_{j}$. Using this, it is not hard to show (see for example [5]) that $\bigcup_{i} \mathfrak{M}_{i}$ contains an ascending sequence $\left\{\mathfrak{N}_{i}\right\}$ of factors such that $\mathfrak{N}_{i}$ is of type $I_{q_{i}}$ and $U_{i} \mathfrak{M}_{i}$ $=\bigcup_{j} \mathfrak{R}_{j}$. Thus $\mathfrak{A}$ is UHF of type $\left\{q_{i}\right\}$.

We now prove a sequence of lemmas, the essence of which is the statement: if $\mathfrak{M}$ is a $C^{*}$-algebra acting on a Hilbert space $\mathfrak{S}$ and if $\left\{e_{i j}: i, j=1, \cdots, n\right\}$ is a family of matrix units acting on $\mathfrak{F}$, and if this family can be approximated in the uniform topology by operators in $\mathfrak{M}$, it can be approximated in the uniform topology by matrix units in $\mathfrak{M}$ (see 1.10). In our applications of these lemmas $\mathfrak{M}$ will be a factor of type $I_{q}$, however it does not seem to simplify the proofs to assume this, since we require inequalities which are independent of $q$.

Lemma 1.6. Let $\epsilon>0$. There is a $\gamma(\epsilon)=\gamma>0$ such that if $\mathfrak{M}$ is a $C^{*}$-algebra acting on a Hilbert space $\mathfrak{S}$, if $E$ is a projection on $\mathfrak{S}$ and if there is an $A \in \mathfrak{M}$ with $\|E-A\|<\gamma$ then there is a projection $F \in \mathfrak{M}$ with $\|E-F\|<\epsilon$.

Proof. Since $\left\|\left(A+A^{*}\right) / 2-E\right\| \leqq\left(\|A-E\|+\left\|A^{*}-E\right\|\right) / 2$ we can assume that $A$ is self-adjoint.

$$
\begin{aligned}
\left\|A^{2}-A\right\| & \leqq\left\|A^{2}-A E\right\|+\|A E-E\|+\|E-A\| \\
& \leqq\left\|A^{2}-A E-E A+E\right\|+\|E A-E\|+\gamma+\gamma \\
& \leqq\|A-E\|^{2}+3 \gamma \leqq \gamma^{2}+3 \gamma .
\end{aligned}
$$

This implies that $\sigma(A)$, the spectrum of $A$, is contained in $[-\delta, \delta]$ $\cup[1-\delta, 1+\delta]$ where $\delta$ is a positive number which can be chosen arbitrarily small by choosing $\gamma$ sufficiently small. We suppose that $\delta<1 / 2$. Let $f$ be the function defined by $f([-\delta, \delta])=0, f([1-\delta, 1+\delta])=1$. Then $f(A) \in \mathfrak{M}, f(A)$ is a projection and

$$
\|f(A)-E\| \leqq\|f(A)-A\|+\|A-E\| \leqq \delta+\gamma .
$$

For a suitable choice of $\gamma<\epsilon / 2, \delta$ can be chosen less than $\epsilon / 2$, and the proof is complete.

Lemma 1.7. If $\epsilon>0$ and if $n$ is a positive integer, there is a $\delta(\epsilon, n)=\delta>0$ with the property that if $\mathfrak{M}$ is a $C^{*}$-algebra and if $\left\{E_{i}: i=1, \cdots, n\right\}$ is a family of projections in $\mathfrak{M}$ with $\left\|E_{i} E_{j}\right\|<\delta$ for $i \neq j$, then there is an orthogonal family $\left\{E_{i}^{\prime}: i=1, \cdots, n\right\}$ of projections in $\mathfrak{M}$, with $\left\|E_{i}^{\prime}-E_{i}\right\|<\epsilon$. 
Proof. We use induction on $n$. If $n=1$ the proof is trivial. Suppose the lemma is true for all $n \leqq r$. For any $\epsilon, 1 / 3>\epsilon>0$, we let $\gamma(\epsilon)$ be the positive number determined by 1.6. We suppose that $\gamma(\epsilon)<\epsilon$, and we define

$$
\delta(\epsilon, r+1)=\min \{1 / 3, \gamma(\epsilon) / 6 r, \delta(\gamma(\epsilon) / 6 r, r)\}
$$

where the right hand side is already defined by the inductive assumption.

Suppose we are given a family $\left\{E_{i}: i=1, \cdots, r+1\right\}$ satisfying the hypothesis of 1.7. By the inductive assumption we can choose an orthogonal family $\left\{E_{i}^{\prime}: i=1, \cdots, r\right\}$ of projections in $\mathfrak{M}$ with $\left\|E_{i}^{\prime}-E_{i}\right\|<\gamma(\epsilon) / 6 r$ for $i=1, \cdots, r$. Let $F=\sum_{i=1}^{r} E_{i}^{\prime}$. Then

$$
\begin{aligned}
\left\|E_{r+1}-(I-F) E_{r+1}(I-F)\right\| & =\left\|E_{r+1}-E_{r+1}-F E_{r+1} F+F E_{r+1}+E_{r+1} F\right\| \\
& \leqq 3\left\|F E_{r+1}\right\| \\
& \leqq 3 \sum_{i=1}^{r}\left\|E_{i}^{\prime} E_{r+1}\right\| \\
& <3 \sum_{i=1}^{r}\left(\left\|E_{i} E_{r+1}\right\|+\gamma(\epsilon) / 6 r\right) \\
& \leqq \gamma(\epsilon) .
\end{aligned}
$$

By our choice of $\gamma(\epsilon)$ we can choose a projection $E_{r+1}^{\prime}$ in the (commutative) $C^{*}$-algebra generated by $E_{i}^{\prime}, i=1, \cdots, r, I$, and $(I-F) E_{r+1}(I-F)$, with $\left\|E_{r+1}^{\prime}-E_{r+1}\right\|<\epsilon$. Hence $\left\|E_{i}^{\prime}-E_{i}\right\|<\epsilon$ for $i=1, \cdots, r+1$. Also $E_{r+1}^{\prime} E_{i}^{\prime}$ is a projection for $i=1, \cdots, r$, and

$$
\begin{aligned}
\left\|E_{r+1}^{\prime} E_{i}^{\prime}\right\| & <\left\|E_{r+1} E_{i}^{\prime}\right\|+\epsilon \\
& \leqq\left\|E_{r+1} E_{i}\right\|+\epsilon+\epsilon \\
& \leqq 1 / 3+1 / 3+1 / 3=1 .
\end{aligned}
$$

Thus $E_{r+1}^{\prime} E_{i}^{\prime}=0$ for $i=1, \cdots, r$ and $\left\{E_{i}^{\prime}: i=1, \cdots, r+1\right\}$ is an orthogonal family of projections in $\mathfrak{M}$.

LemMA 1.8. If $\left\{E_{i}: i=1, \cdots, n\right\}$ and $\left\{F_{i}: i=1, \cdots, n\right\}$ are each orthogonal families of projections in a $C^{*}$-algebra $\mathfrak{M}$, and if $\left\|E_{i}-F_{i}\right\|<1$, then there is a partial isometry $W$ in $\mathfrak{M}$ such that $E_{i} W F_{i}$ is a partial isometry from $F_{i}$ to $E_{i}$. If $\epsilon>0$ there is a $\delta(\epsilon, n)=\delta>0$ such that if $\left\|E_{i}-F_{i}\right\|<\delta$ then $W$ can be chosen so that $\left\|E_{i}-E_{i} W F_{i}\right\|<\epsilon$. If $\sum_{i} E_{i}=I$ then $W$ can be chosen so that $\|I-W\|<\epsilon$.

Proof. We have the inequalities $\left\|E_{i}-E_{i} F_{i} E_{i}\right\|,\left\|F_{i}-F_{i} E_{i} F_{i}\right\| \leqq\left\|E_{i}-F_{i}\right\|$ $<1$. Let $\gamma$ be a number such that $\left\|E_{i}-F_{i}\right\|<\gamma<1$. Let $f$ be the function defined by $f(x)=0$ if $x \leqq(1-\gamma) / 2, f(x)=1 / x$ if $x \geqq 1-\gamma, f$ is linear on $[(1-\gamma) / 2,1-\gamma]$. If $\rho$ is a pure state of the (commutative) $C^{*}$-algebra generated by $I, E_{i}$ and $E_{i} F_{i} E_{i}$, then $\rho\left(E_{i}\right) \in\{0,1\}$. If $\rho\left(E_{i}\right)=0$ (resp. 1) then since 
$0 \leqq E_{i} F_{i} E_{i} \leqq E_{i}$ (resp. $\left.0 \leqq E_{i}-E_{i} F_{i} E_{i} \leqq \gamma\right)$, we have $\rho\left(E_{i} F_{i} E_{i}\right)=0$ (resp. $\in[1-\gamma, 1]$ ). Since $\rho$ is multiplicative,

$$
\rho\left(E_{i} F_{i} E_{i} f\left(E_{i} F_{i} E_{i}\right)\right)=\rho\left(E_{i} F_{i} E_{i}\right) f\left(\rho\left(E_{i} F_{i} E_{i}\right)\right)=\rho\left(E_{i}\right),
$$

and if we let $G_{i}=\left(f\left(E_{i} F_{i} E_{i}\right)\right)^{1 / 2}$ then since (1) holds for each $\rho, E_{i}=E_{i} F_{i} E_{i} G_{i}^{2}$ $=G_{i} E_{i} F_{i} E_{i} G_{i}$. If we let $H_{i}=\left(f\left(F_{i} E_{i} F_{i}\right)\right)^{1 / 2}$ then by a similar argument, $F_{i}=H_{i}^{2} F_{i} E_{i} F_{i}$. Let $W_{i}=E_{i} G_{i} F_{i}$. Then $W_{i} W_{i}^{*}=E_{i} G_{i} F_{i} G_{i} E_{i}=E_{i}$ and

$$
\begin{aligned}
W_{i}^{*} W_{i} & =F_{i} G_{i} E_{i} G_{i} F_{i} \\
& =H_{i}^{2} F_{i} E_{i} F_{i} E_{i} G_{i}^{2} F_{i} \\
& =H_{i}^{2} F_{i} E_{i} F_{i}=F_{i} .
\end{aligned}
$$

Let $W=\sum_{i} W_{i} . E_{i} W F_{i}=W_{i}$ is a partial isometry from $F_{i}$ to $E_{i}$ and $W$ is a partial isometry from $\sum_{i} F_{i}$ to $\sum_{i} E_{i}$.

Given a positive $\epsilon$, let $\delta$ be the minimum of $\{1 / 2, \epsilon / 4 n\}$. If $\left\|E_{i}-F_{i}\right\|<\delta$ then we choose $\gamma$ in the first part of the proof to be equal to $\delta$. We have

$$
\begin{aligned}
\left\|W_{i}-E_{i}\right\| & \leqq\left\|W_{i}-G_{i}\right\|+\left\|G_{i}-E_{i}\right\| \\
& =\left\|G_{i} F_{i}-G_{i} E_{i}\right\|+\left\|G_{i}-E_{i}\right\| \\
& \leqq\left\|G_{i}\right\|\left\|F_{i}-E_{i}\right\|+\left\|G_{i}-E_{i}\right\| .
\end{aligned}
$$

If $\rho$ is as above, then $\left|\rho\left(G_{i}-E_{i}\right)\right| \leqq\left|\rho\left(G_{i}^{2}\right)-\rho\left(E_{i}\right)\right|<(1-\delta)^{-1}-1=\delta /(1-\delta)$ and $\left|\rho\left(G_{i}\right)\right| \leqq\left|\rho\left(G_{i}^{2}\right)\right|<1 /(1-\delta)$. Consequently $\left\|G_{i}-E_{i}\right\|<\delta /(1-\delta)$ and $\left\|G_{i}\right\|<1 /(1-\delta)$ and so $\left\|W_{i}-E_{i}\right\|<2 \delta /(1-\delta) \leqq 4 \delta \leqq \epsilon / n$. If $\sum_{i} E_{i}=I$, then

$$
\begin{aligned}
\|W-I\| & =\left\|\sum_{i}\left(W_{i}-E_{i}\right)\right\| \\
& \leqq \sum_{i}\left\|W_{i}-E_{i}\right\|<\epsilon .
\end{aligned}
$$

Lemma 1.9. Let $\epsilon>0$. There is $a \delta(\epsilon)=\delta>0$ with the following property: Let $\mathfrak{M}$ be a $C^{*}$-algebra acting on a Hilbert space $\mathfrak{S}$. Let $E_{1}$ and $E_{2}$ (resp. $F_{1}$ and $F_{2}$ ) be orthogonal projections in $\mathfrak{M}$ (resp. operating on $\mathfrak{S}$ ). Suppose that $\left\|E_{i}-F_{i}\right\|<\delta, i=1,2$, and suppose that there is a partial isometry $V$ from $F_{1}$ to $F_{2}$ and an $A \in \mathfrak{M}$ such that $\|V-A\|<\delta$. Then there is a partial isometry $U \in \mathfrak{M}$ from $E_{1}$ to $E_{2}$ and $\|V-U\|<\epsilon$.

Proof. Let $\delta=\min \{1 / 32, \epsilon / 75\}$. We have

$$
\begin{aligned}
\left\|E_{2}-E_{2} A A^{*} E_{2}\right\| & \leqq\left\|E_{2}-A A^{*}\right\| \\
& \leqq\left\|E_{2}-F_{2}\right\|+\left\|F_{2}-A A^{*}\right\| \\
& <\delta+\left\|V V^{*}-V A^{*}\right\|+\left\|V A^{*}-A A^{*}\right\| \\
& <\delta+\delta+\delta(1+\delta)<4 \delta<1 .
\end{aligned}
$$

$E_{2} A A^{*} E_{2}$ is positive and lives on $E_{2}$. By spectral theory, there is an $X \in \mathfrak{M}$ such that 


$$
\begin{aligned}
E_{2} & =X E_{2} A A^{*} E_{2} X \\
\left\|X-E_{2}\right\| & \leqq\left\|X^{2}-E_{2}\right\|<4 \delta /(1-4 \delta)<8 \delta .
\end{aligned}
$$

Let $W=X E_{2} A$. Then $W W^{*}=E_{2}$ and $W^{*} W$ is a projection in $\mathfrak{M}$. Also

$$
\begin{aligned}
\left\|W^{*} W-E_{1}\right\| \leqq & \left\|W^{*} W-V^{*} E_{2} X^{2} E_{2} A\right\|+\left\|V^{*} E_{2} X^{2} E_{2} A-V^{*} E_{2} X^{2} E_{2} V\right\| \\
& \quad+\left\|V^{*} E_{2} X^{2} E_{2} V-F_{1}\right\|+\left\|F_{1}-E_{1}\right\| \\
\leqq & \left\|V^{*}-A^{*}\right\|\left\|X^{2}\right\|\|A\|+\|A-V\|\left\|X^{2}\right\|+\left\|E_{2} X^{2} E_{2}-F_{2}\right\|+\delta \\
< & \delta(1+8 \delta)(1+\delta)+\delta(1+8 \delta)+\left\|E_{2} X^{2} E_{2}-E_{2}\right\|+\delta+\delta \\
\leqq & 8 \delta+\left\|X^{2}-E_{2}\right\|<16 \delta \leqq 1 / 2 .
\end{aligned}
$$

By the $\delta$ chosen in the proof of 1.8 , we see that there is a partial isometry $W_{1} \in \mathfrak{M}$ from $E_{1}$ to $W^{*} W$ and

$$
\left\|W^{*} W-W_{1}\right\|<64 \delta .
$$

Let $U=W W_{1}$. Then

$$
\begin{aligned}
\|V-U\| \leqq & \|V-W\|+\left\|W-W W_{1}\right\| \\
\leqq & \left\|V-E_{2} V\right\|+\left\|E_{2} V-X E_{2} V\right\|+\left\|X E_{2} V-X E_{2} A\right\| \\
& \quad+\left\|W^{*} W-W_{1}\right\| \\
< & \delta+8 \delta+\delta(1+8 \delta)+64 \delta \leqq 75 \delta \leqq \epsilon .
\end{aligned}
$$

$U$ is a partial isometry from $E_{1}$ to $E_{2}$, and the proof is complete.

Lemma 1.10. For every $\epsilon>0$ and every positive integer $n$ there is a $\delta(\epsilon, n)$ $=\delta>0$ with the following property. Let $\mathfrak{M}$ be a $C^{*}$-algebra acting on a Hilbert space $\mathfrak{S}$, let $\left\{e_{i j}: i, j=1, \cdots, n\right\}$ be a family of matrix units acting on $\mathfrak{S}$. If there are $A_{i j} \in \mathfrak{M}$ with $\left\|e_{i j}-A_{i j}\right\|<\delta$ then there are matrix units $f_{i j} \in \mathfrak{M}$ with $\left\|e_{i j}-f_{i j}\right\|<\epsilon$.

Proof. By 1.6 we suppose without loss of generality that $A_{i i}$ is a projection. For $i \neq j$,

$$
\begin{aligned}
\left\|A_{i i} A_{j j}\right\| & \leqq\left\|A_{i i} A_{j j}-e_{i i} A_{j j}\right\|+\left\|e_{i i} A_{j j}-e_{i i} e_{j j}\right\| \\
& \leqq\left\|A_{i i}-e_{i i}\right\|+\left\|A_{j j}-e_{j j}\right\| .
\end{aligned}
$$

By 1.7 we can assume without loss of generality that $\left\{A_{i i}\right\}$ is an orthogonal family of projections and by 1.9 that for $i \neq j, A_{i j}$ is a partial isometry from $A_{j j}$ to $A_{i i} \cdot \sum_{i} A_{i i}$ is a projection and

$$
\left\|I-\sum_{i} A_{i i}\right\| \leqq \sum_{i}\left\|e_{i i}-A_{i i}\right\|
$$

which for $\delta<1 / n$ is less than 1 . In this case $\sum_{i} A_{i i}=I$. Let $f_{i j}=A_{1 i}^{*} A_{1 j}$. If $\delta<\min (1 / n, \epsilon / 2)$ then $\left\{f_{i j}\right\}$ is a family of matrix units and 


$$
\begin{aligned}
\left\|f_{i j}-e_{i j}\right\| & \leqq\left\|A_{1 i}^{*} A_{1 j}-A_{1 i}^{*} e_{1 j}\right\|+\left\|A_{1 i}^{*} e_{1 j}-e_{i j}\right\| \\
& <2 \delta \leqq \epsilon .
\end{aligned}
$$

This proof is complete.

Lemma 1.11. For every positive integer $n$ there is an $\epsilon>0$ with the following property. Let $\mathfrak{M}$ be a factor of type $\mathrm{I}_{q}$ acting on a Hilbert space $\mathfrak{S}$, let $\left\{e_{i j}: i, j=1, \cdots, n\right\}$ be a family of matrix units acting on $\mathfrak{S}$. If in addition there are $A_{i j} \in \mathfrak{M}$ with $\left\|e_{i j}-A_{i j}\right\|<\epsilon$ then $n \mid q$.

Proof. By 1.10 it is sufficient to prove 1.11 when $\left\{A_{i j}\right\}$ is a family of matrix units. Assuming this, recall that $I=\sum_{i} A_{i i}$. Hence if $\operatorname{Dim}(\cdot)$ is the dimension function for $\mathfrak{M}, 1=\operatorname{Dim}\left(\sum_{i} A_{i i}\right)=n \operatorname{Dim}\left(A_{i i}\right)$ for any $i$, and $\operatorname{Dim}\left(A_{i i}\right)=1 / n$. This implies $n \mid q$.

We prove two theorems, which are the main results of this section. The first theorem is a classification of UHF algebras under ${ }^{*}$-isomorphisms.

THEOREM 1.12. If $\mathfrak{A}$ and $\mathfrak{B}$ are UHF algebras of type $\left\{p_{i}\right\}$ and $\left\{r_{i}\right\}$ respectively, then $\mathfrak{A}$ is *-isomorphic to $\mathfrak{B}$ if and only if $f\left(\left\{p_{i}\right\}\right)=f\left(\left\{r_{i}\right\}\right)$, where $f$ is defined in 1.3 .

Proof. Suppose $f\left(\left\{p_{i}\right\}\right)=f\left(\left\{r_{i}\right\}\right)$. The $q_{k}$ defined in 1.4 with the sequence $\left\{p_{i}\right\}$ is the same as the $q_{k}$ defined with the sequence $\left\{r_{i}\right\}$ for $k=1,2, \cdots$. By $1.5 \mathfrak{A}$ and $\mathfrak{B}$ are both of type $\left\{q_{i}\right\}$ and by $1.2 \mathfrak{A}$ and $\mathfrak{B}$ are ${ }^{*}$-isomorphic.

If $\mathfrak{A}$ and $\mathfrak{B}$ are ${ }^{*}$-isomorphic, it will follow from the second statement of 1.13 that $f\left(\left\{p_{i}\right\}\right)=f\left(\left\{r_{i}\right\}\right)$. A direct proof could be given, using 1.11.

In the next theorem we state a necessary and sufficient condition for a $C^{*}$-algebra to be either UHF or a factor of type $\mathrm{I}_{n}$. This condition is analogous to one of the definitions of hyperfinite factors of type $\mathrm{II}_{1}[4]$. We also find a new definition for $f\left(\left\{p_{i}\right\}\right)$.

Theorem 1.13. Let $\mathfrak{A}$ be a $C^{*}$-algebra. $\mathfrak{A}$ is either a $U H F$ algebra or a factor of type $\mathrm{I}_{n}$ if and only if the following conditions are satisfied.

(1) $\mathfrak{A}$ has a countable dense subset.

(2) If $A_{1}, \cdots, A_{n} \in \mathfrak{A}$ and if $\epsilon>0$ there is a positive integer $r$ and a factor $\mathfrak{M} \subset \mathfrak{A}$ of type $\mathrm{I}_{r}$ and $B_{1}, \cdots, B_{n} \in \mathfrak{M}$ such that $\left\|A_{i}-B_{i}\right\|<\epsilon$.

If we define for a $C^{*}$-algebra $\mathfrak{A}$ and a prime number $x$

$$
f(\mathfrak{A}, x)=\sup \left\{j: \mathfrak{M} \text { is a factor of type } \mathrm{I}_{n}, \mathfrak{M} \subset \mathfrak{A}, x^{j} \mid n\right\}
$$

then if $\mathfrak{A}$ is a UHF algebra of type $\left\{p_{i}\right\}, f\left(\left\{p_{i}\right\}\right)(\cdot)=f(\mathfrak{A}, \cdot)$.

Proof. Suppose $\mathfrak{A}$ satisfies (1) and (2). Let $\left\{D_{i}: i=1,2, \cdots\right\}$ be a countable dense subset of the sphere of radius $1 / 2$ about the origin of $\mathfrak{A}$. We construct by induction an ascending sequence of factors $\left\{\mathfrak{M}_{i}\right\}, \mathfrak{M}_{i}$ of type $\mathrm{I}_{p_{i}}$ for some positive integer $p_{i}, \mathfrak{M}_{i} \subset \mathfrak{A}$, and such that there are $B_{k} \in \mathfrak{M}_{i}, k=1, \cdots, i$ with $\left\|D_{k}-B_{k}\right\| \leqq 2^{-i}$. The existence of $\mathfrak{M}_{1}$ is a direct 
consequence of (2). Suppose $\mathfrak{M}_{i}$ has been chosen and satisfies the above conditions for $i=1, \cdots, n$. We construct $\mathfrak{M}_{n+1}$.

Let $\left\{e_{s t}\right\}$ be a family of matrix units for $\mathfrak{M}_{n}$, let $\epsilon=2^{-n-5} p_{n}^{-2}$. By (2) and 1.10 we can find a factor $\mathfrak{M} \subset \mathfrak{A}, \mathfrak{M}$ of type $\mathrm{I}_{p_{n+1}}$, for some positive integer $p_{n+1}$ such that there are matrix units $f_{s t} \in\{\mathfrak{M}\}$ with $\left\|e_{s t}-f_{s t}\right\|<\epsilon, s, t$ $=1, \cdots, p_{n}$, and $B_{k}^{\prime} \in \mathfrak{M}$ with $\left\|D_{k}-B_{k}^{\prime}\right\|<\epsilon, k=1, \cdots, n+1$. By 1.8 and the choice of $\delta$ in the proof of 1.8 , there is a partial isometry $W \in \mathfrak{A}$ from $f_{11}$ to $e_{11}$ with $\left\|e_{11}-W\right\|<4 \epsilon$. Let $\mathfrak{M}_{n+1}$ be the $C^{*}$-algebra generated by $\mathfrak{M}_{n}$ and $W f_{11} \mathfrak{M} f_{11} W^{*} . \mathfrak{M}_{n+1}$ is isomorphic to the tensor product of $\mathfrak{M}_{n}$ and $f_{11} \mathfrak{M} f_{11}$, and so is a factor of type $\mathrm{I}_{p_{n+1}}$, and $\mathfrak{M}_{n} \subset \mathfrak{M}_{n+1} \subset \mathfrak{A}$. Let

$$
B_{k}=\sum_{s t} e_{s 1} W f_{1 s} B_{k}^{\prime} f_{t 1} W^{*} e_{1 t} \text {. }
$$

Then $B_{k} \in \mathfrak{M}_{n+1}$ and

$$
\begin{aligned}
\left\|D_{k}-B_{k}\right\| & \leqq\left\|D_{k}-B_{k}^{\prime}\right\|+\left\|B_{k}^{\prime}-B_{k}\right\| \\
& \leqq \epsilon+p_{n}^{2} \max _{s, t}\left\|f_{s s} B_{k}^{\prime} f_{t t}-e_{s 1} W f_{1 s} B_{k}^{\prime} f_{t 1} W^{*} e_{1 t}\right\| .
\end{aligned}
$$

However

$$
\begin{aligned}
& \left\|f_{s 8} B_{k}^{\prime} f_{t t}-e_{s 1} W f_{18} B_{k}^{\prime} f_{t 1} W^{*} e_{1 t}\right\| \\
& \quad \leqq\left\|f_{88} B_{k}^{\prime} f_{t t}-f_{88} B_{k}^{\prime} f_{t 1} W^{*} e_{1 t}\right\|+\left\|f_{88} B_{k}^{\prime} f_{t 1} W^{*} e_{1 t}-e_{s 1} W f_{1 s} B_{k}^{\prime} f_{t 1} W^{*} e_{1 t}\right\| \\
& \quad \leqq\left\|f_{t t}-f_{t 1} W^{*} e_{1 t}\right\|+\left\|f_{s 8}-e_{s 1} W f_{18}\right\| \\
& \quad \leqq\left\|f_{1 t}-W^{*} e_{1 t}\right\|+\left\|f_{81}-e_{81} W\right\| \\
& \quad \leqq\left\|f_{1 t}-e_{1 t}\right\|+\left\|e_{1 t}-W^{*} e_{1 t}\right\|+\left\|f_{s 1}-e_{s 1}\right\|+\left\|e_{s 1}-e_{s 1} W\right\| \\
& \quad \leqq \epsilon+4 \epsilon+\epsilon+4 \epsilon .
\end{aligned}
$$

Thus

$$
\left\|D_{k}-B_{k}\right\| \leqq \epsilon+10 p_{n}^{2} \epsilon \leqq 2^{-n-1}
$$

for $k=1, \cdots, n+1$, and $\mathfrak{M}_{n+1}$ has been constructed and has the required properties. We can suppose that $\mathfrak{M}_{i}$ has been constructed and has the required properties for $i=1,2, \ldots$. The closure of $U_{i} \mathfrak{M}_{i}$ contains $\left\{D_{i}: i=1,2, \cdots\right\}$ and so coincides with $\mathfrak{A}$. If $p_{i} \rightarrow \infty$ as $i \rightarrow \infty$ then $\mathfrak{A}$ is UHF. If $p_{i} \rightarrow \infty$ as $i \rightarrow \infty$, choose a $j$ such that $p_{j}=p_{j+1}=\cdots$. Then $\mathfrak{M}_{j}$ is dense in $\mathfrak{A}, \mathfrak{M}_{j}$ equals $\mathfrak{A}$ and $\mathfrak{A}$ is a factor of type $\mathrm{I}_{r_{j}}$. This proves the first statement of 1.13 .

We prove the second statement. Let $\mathfrak{A}$ be a UHF algebra of type $\left\{p_{i}\right\}$, let $N=\left\{n: \mathfrak{M}\right.$ is a factor of type $\left.\mathrm{I}_{n}, \mathfrak{M} \subset \mathfrak{A}\right\}$. Let $x$ be a prime number. We must show that $f\left(\left\{p_{i}\right\}\right)(x)=\sup \left\{j\right.$ : there is an $n \in N$ with $\left.x^{j} \mid n\right\}$. Suppose $n \in N$. By 1.11 and 1.1 formula (4) there is an $i$ such that $n \mid p_{i}$. Thus 


$$
\begin{aligned}
& \max \left\{j: x^{j} \mid n\right\} \leqq f\left(\left\{p_{i}\right\}\right)(x), \\
& \sup \left\{j: \text { there is an } n \in N \text { with } x^{j} \mid n\right\} \leqq f\left(\left\{p_{i}\right\}\right)(x) .
\end{aligned}
$$

The converse inequality follows directly from the definition of $f\left(\left\{p_{i}\right\}\right)$ and the fact that $p_{i} \in N$. This completes the proof of 1.13 .

In $\S 5$ we will construct a $C^{*}$-algebra which is related to the investigations of this section.

2. Pure states and the pure state space. Let $\mathfrak{A}$ be a UHF algebra generated by factors $\mathfrak{M}_{n}$ of type $\mathrm{I}_{p_{n}}$, let $\left\{e_{i j}^{n}: i, j=1, \cdots, p_{n}\right\}$ be a family of matrix units for $\mathfrak{M}_{n}$. If $f$ is a linear functional on $\mathfrak{A}$, then $f$ is uniquely determined by the numbers $a_{i j}^{n}=f\left(e_{i j}^{n}\right)$. We determine in 2.4 necessary and sufficient conditions in terms of the $a_{i j}^{n}$ that $f$ be a state (resp. pure state) of $\mathfrak{A}$. If $f$ is a state of $\mathfrak{A}$ then $f$ is pure if and only if for each positive integer $n$ there is an integer $r$ greater than $n$ such that if $g$ is a linear functional on $\mathfrak{M}_{r}$, and if $f \mid \mathfrak{M}_{r} \geqq g \geqq 0$ then $g \mid \mathfrak{M}_{n}$ is approximately a scalar multiple of $f \mid \mathfrak{M}_{n}$. First we prove two lemmas dealing with a more general situation. If $T$ is a subset of a metric space $T^{\prime}$, if $\epsilon>0$, we say that $T$ is $\epsilon$-dense in $T^{\prime}$ if for every $t^{\prime}$ in $T^{\prime}$ there is a $t$ in $T$ with $d\left(t^{\prime}, t\right)<\epsilon$.

Lemma 2.1. Let $\mathfrak{A}$ be a $C^{*}$-algebra, let $\left\{\mathfrak{M}_{n}: n \in N\right\}$ be a net (directed by inclusion, $N$ a set of indices) of finite dimensional self-adjoint linear subspaces $\mathfrak{M}_{n}$ of $\mathfrak{A}$ which contain $I$. Assume that $\mathfrak{A}$ is the closure of the union of the $\mathfrak{M}_{n}$. (Such a net always exists.) Let $\omega$ be a state of $\mathfrak{A} . \omega$ is pure if for each $n$ in $N$ and each positive number $\epsilon$ there is an $r_{1}(n, \epsilon)=r$ in $N$ with $r \geqq n$ and such that there exists an $\epsilon$-dense subset $T$ of $\left\{a \tau: a \in[0,1], \tau\right.$ is a state of $\mathfrak{M}_{r}$ and $\left.\omega \mid \mathfrak{M}_{r} \geqq a \tau\right\}$

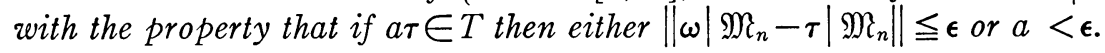

Proof. Suppose $\omega$ is not pure. Then $\omega=2^{-1} \tau_{1}+2^{-1} \tau_{2}$, where $\tau_{1}$ and $\tau_{2}$ are distinct states of $\mathfrak{A} . \tau_{1}$ and $\omega$ are also distinct states of $\mathfrak{A}$, and since $\bigcup_{n} \mathfrak{M}_{n}$ is dense in $\mathfrak{A}, \omega \mid \mathfrak{M}_{n}$ and $\tau_{1} \mid \mathfrak{M}_{n}$ are distinct states of $\mathfrak{M}_{n}$ for some $n$ in $N$. Let $\epsilon=\min \left\{1 / 4,\left\|\omega\left|\mathfrak{M}_{n}-\tau_{1}\right| \mathfrak{M}_{n}\right\| / 5\right\}$, let $r$ be in $N$ with $r \geqq n$, let $T$ be an $\epsilon$-dense subset of $\left\{a \tau: a \in[0,1], \tau\right.$ is a state of $\mathfrak{M}_{r}$ and $\left.\omega \mid \mathfrak{M}_{r} \geqq a \tau\right\}$. Since $\omega \mid \mathfrak{M}_{r}$ $\geqq 2^{-1} \tau_{1} \mid \mathfrak{M}_{r}$, there is an $a \tau$ in $T$ with $\left\|2^{-1} \tau_{1} \mid \mathfrak{M}_{r}-a \tau\right\|<\epsilon$. This implies $\left|2^{-1}-a\right|$ $<\epsilon, a>\epsilon$, and

$$
\begin{aligned}
\left\|\omega\left|\mathfrak{M}_{n}-\tau\right| \mathfrak{M}_{n}\right\| & \geqq\left\|\omega\left|\mathfrak{M}_{n}-\tau_{1}\right| \mathfrak{M}_{n}\right\|-\left\|\tau_{1} \mid \mathfrak{M}_{r}-2 a \tau\right\|-\|2 a \tau-\tau\| \\
& >5 \epsilon-2 \epsilon-2 \epsilon=\epsilon .
\end{aligned}
$$

Thus for this $n$ and $\epsilon$, there is no $r$ with the properties of the lemma, and the proof is complete.

Lemma 2.2. Let $\mathfrak{A},\left\{\mathfrak{M}_{n}: n \in N\right\}$, and $\omega$ be as in 2.1. $\omega$ is pure only if for each $n$ in $N$ and each positive number $\epsilon$ there is an $r_{2}(n, \epsilon)=r$ in $N$ with $r \geqq n$ and such that if $q \in N$ and if $q \geqq r$, and if

$$
\omega \mid \mathfrak{M}_{q}=\sum_{k} \alpha_{k} \tau_{k}
$$


where $\tau_{k}$ is a state of $\mathfrak{M}_{q}$ and $\left\{\alpha_{k}\right\}$ is a finite convex family, and if we let

$$
K_{q}=\left\{k:\left\|\omega\left|\mathfrak{M}_{n}-\tau_{k}\right| \mathfrak{M}_{n}\right\|>\epsilon\right\}
$$

then

$$
\sum\left\{\alpha_{k}: k \in K_{q}\right\}<\epsilon .
$$

Proof. We suppose that for some $n$ and some positive $\epsilon$ such an $r$ cannot be found. For each $q \geqq n$ in some cofinal subset $Q$ of $N$, there is a decomposition of $\omega \mid \mathfrak{M}_{q}$ as a finite convex sum of restrictions to $\mathfrak{M}_{q}$ of states $\left\{\tau_{k q}: k=1, \cdots, t_{q}\right\}$ of $\mathfrak{A}$,

$$
\omega\left|\mathfrak{M}_{q}=\sum_{k} \alpha_{k q} \tau_{k q}\right| \mathfrak{M}_{q}
$$

and if $K_{q}$ is defined as in the statement of the theorem, $\sum\left\{\alpha_{k q}: k \in K_{q}\right\} \geqq \epsilon$. The dual $\mathfrak{M}_{n}^{*}$ to the topological vector space $\mathfrak{M}_{n}$ is a finite dimensional topological vector space, so the weak topology and the norm topology on $\mathfrak{M}_{n}^{*}$ are the same. Consequently there is a finite subset $\left\{A_{i}: i=1, \cdots, s\right\}$ of nonzero elements of $\mathfrak{M}_{n}$ such that if $f \in \mathfrak{M}_{n}^{*}$ and $\left|f\left(A_{i}\right)\right| \leqq 1, i=1, \cdots, s$ then $\|f\| \leqq \epsilon$. $\omega\left|\mathfrak{M}_{n}-\tau_{k q}\right| \mathfrak{M}_{n} \in \mathfrak{M}_{n}^{*}$ and so

$$
K_{q}=\bigcup\left\{K(q, i) \cap K_{q}: i=1, \cdots, 2 s\right\}
$$

where

$$
\begin{aligned}
K(q, 2 i) & =\left\{k:-\omega\left(A_{i}\right)+\tau_{k q}\left(A_{i}\right)>1\right\}, & & i=1, \cdots, s \\
K(q, 2 i-1) & =\left\{k: \omega\left(A_{i}\right)-\tau_{k q}\left(A_{i}\right)>1\right\}, & & i=1, \cdots, s .
\end{aligned}
$$

Since $\sum\left\{\alpha_{k q}: k \in K_{q}\right\} \geqq \epsilon$ there is an $i(q) \in\{1, \cdots, 2 s\}$ such that

$$
a_{q}=\sum\left\{\alpha_{k q}: k \in K(q, i(q))\right\} \geqq \epsilon / 2 s
$$

where the above equation defines $a_{q}$. Let

$$
\begin{aligned}
\tau_{q}^{\prime} & =a_{q}^{-1} \sum\left\{\alpha_{k q} \tau_{k q}: k \in K(q, i(q))\right\}, \\
\tau_{q}^{\prime \prime} & =\left(1-a_{q}\right)^{-1} \sum\left\{\alpha_{k q} \tau_{k q}: k \notin K(q, i(q)), 1 \leqq k \leqq t_{q}\right\} .
\end{aligned}
$$

Then

$$
\omega\left|\mathfrak{M}_{q}=a_{q} \tau_{q}^{\prime}\right| \mathfrak{M}_{q}+\left(1-a_{q}\right) \tau_{q}^{\prime \prime} \mid \mathfrak{M}_{q}
$$

and letting $j=i(q) / 2$ if $i(q)$ is even, $(i(q)+1) / 2$ if $i(q)$ is odd,

$$
\begin{aligned}
\left\|\omega\left|\mathfrak{M}_{n}-\tau_{q}^{\prime}\right| \mathfrak{M}_{n}\right\| & \geqq\left\|A_{j}\right\|^{-1}\left|\omega\left(A_{j}\right)-\tau_{q}^{\prime}\left(A_{j}\right)\right| \\
& \geqq\left\|A_{j}\right\|^{-1} .
\end{aligned}
$$

The states of $\mathscr{A}$ are $w^{*}$-compact, so we can choose a subnet $\left\{q_{m}: m \in M\right\}$ of $Q$, where $M$ is some directed set, such that 


$$
\begin{gathered}
\tau_{q_{m}}^{\prime} \rightarrow \tau^{\prime} \\
\tau_{q_{m}}^{\prime \prime} \rightarrow \tau^{\prime \prime} \\
a_{q_{m}} \rightarrow a
\end{gathered}
$$

where $\tau^{\prime}, \tau^{\prime \prime}$ are states of $\mathfrak{A}$, the convergence in (2) and (3) is in the $w^{*}$ topology, and $a$ is a real number. If $A \in \mathfrak{M}_{q}$,

$$
\begin{aligned}
a \tau^{\prime}(A)+(1-a) \tau^{\prime \prime}(A) & =\lim _{m}\left(a_{q_{m}} \tau_{q_{m}}^{\prime}(A)+\left(1-a_{q_{m}}\right) \tau_{q_{m}}^{\prime \prime}(A)\right) \\
& =\lim _{m} \omega(A)=\omega(A) .
\end{aligned}
$$

Consequently $a \tau^{\prime}+(1-a) \tau^{\prime \prime}=\omega$. We show that this convex sum is not degenerate, and this will complete the proof. By the equivalence of weak and norm topologies in $\mathfrak{M}_{n}^{*}$,

$$
\begin{aligned}
\left\|\omega\left|\mathfrak{M}_{n}-\tau^{\prime}\right| \mathfrak{M}_{n}\right\| & =\left\|\omega\left|\mathfrak{M}_{n}-\left(\underset{m}{\lim } \tau_{q_{m}}^{\prime}\right)\right| \mathfrak{M}_{n}\right\| \\
& =\left\|\omega \mid \mathfrak{M}_{n}-\lim _{m}\left(\tau_{q_{m}}^{\prime} \mid \mathfrak{M}_{n}\right)\right\| \\
& =\lim _{m}\left\|\omega\left|\mathfrak{M}_{n}-\tau_{q_{m}}^{\prime}\right| \mathfrak{M}_{n}\right\| \\
& \geqq \inf \left\{\left\|A_{j}\right\|-1: j=1, \cdots, s\right\}>0 .
\end{aligned}
$$

Thus $\omega \neq \tau^{\prime}$, which implies $a<1$ and $\tau^{\prime} \neq \tau^{\prime \prime}$. By (1) $a>0$ and the proof is complete.

We observe that 2.2 is (stronger than) a converse to 2.1 . Suppose that $\omega$ is pure, that $n \in N$ and that $\epsilon>0$. We show that there is an $r_{1}(n, \epsilon)$ which has the properties of 2.1. In fact, let $r_{1}(n, \boldsymbol{\epsilon})=r_{2}(n, \epsilon)(=r)$. Let $T=\{a \tau: a \in[0,1)$ and $\tau$ is a state of $\mathfrak{M}_{r}$ with $\left.\omega \mid \mathfrak{M}_{r} \geqq a \tau\right\}$. If $a \tau \in T$ then

$$
\omega \mid \mathfrak{M}_{r}=a \tau+(1-a)\left[(1-a)^{-1}\left(\omega \mid \mathfrak{M}_{r}-a \tau\right)\right]
$$

and by 2.2 , either $\left\|\omega\left|\mathfrak{M}_{n}-\tau\right| \mathfrak{M}_{n}\right\| \leqq \epsilon$ or $a<\epsilon$.

Definition 2.3. Let $\mathfrak{R}$ (resp. $\mathfrak{M}$ ) be the algebra of complex $n \times n$ (resp. $q \times q)$ matrices, with matrix units $\left\{e_{i j}^{n}\right\}$ (resp. $\left\{e_{i j}^{q}\right\}$ ). Suppose $n \mid q$. An isomorphism $\theta$ of $\mathfrak{N}$ into $\mathfrak{M}$ is called the standard imbedding [5] if

$$
\theta\left(e_{i j}^{n}\right)=\sum\left\{e_{s t}^{q}:(s, t)=(a n+i, a n+j), a=0, \cdots,(q / n)-1\right\} .
$$

We identify the set of $p_{r} \times p_{r}$ complex matrices with $\mathfrak{M}_{r}$ by means of the matrix units $\left\{e_{i j}^{r}\right\}$. When we speak of an $\epsilon$-dense subset $S$ of a subset of $\mathfrak{M}^{r}$, we shall mean $\epsilon$-dense in the metric which makes the mapping $\phi$ of 6.1 from the normed space $\mathfrak{M}_{r}^{*}$ to the set $\mathfrak{M}_{r}$ an isometry. If $B_{1}$ and $B_{2}$ are positive elements of $\mathfrak{M}_{r}$, then the distance from $B_{1}$ to $B_{2}$ in this metric is trace $\left|B_{1}-B_{2}\right|$ (see the proof of 3.4). 
TheOREM 2.4. Using the notation preceding 2.1, let $A_{n}$ be the tranpose of the matrix $\left(a_{i j}^{n}\right)$. Then $f$ is a state if and only if trace $\left(A_{n}\right)=1$ and $A_{n} \geqq 0$ for each $n$. Suppose that $f$ is a state and that the inclusion $\mathfrak{M}_{n} \rightarrow \mathfrak{M}_{q}$ is the standard imbedding relative to the matrix units $\left\{e_{i j}^{n}\right\}$ and $\left\{e_{i\}}^{q}\right\}$ for each $n$ and $q$ with $q \geqq n$. Then $f$ is pure if for each positive integer $n$ and each positive $\epsilon$ there is an integer $r_{3}(n, \epsilon)=r$ with $r \geqq n$ and such that there exists an $\epsilon$-dense subset $S(n, \epsilon)=S$ of the set $\left\{a B: a \in[0,1]\right.$ and $B$ is a positive $p_{r} \times p_{r}$ matrix with trace $(B)=1$ and $\left.A_{r} \geqq a B\right\}$ with the property that if $a B \in S$ then either

$$
\sup _{i j}\left|a_{i j}^{n}-\sum\left\{b_{t s}:(s, t)=\left(a p_{n}+i, a p_{n}+j\right), a=0, \cdots,\left(p_{r} / p s\right)-1\right\}\right| \leqq \epsilon
$$

or $a<\epsilon$ where $B$ is the matrix $\left(b_{s t}\right)$. Also $f$ is pure only if for each positive integer $n$ and each positive $\epsilon$ there is an integer $r_{4}(n, \epsilon)=r$ with $r \geqq n$ and such that if $q$ is an integer, if $q \geqq r$, if

$$
A_{q}=\sum_{k} \alpha_{k} B_{k}
$$

where $\left\{\alpha_{k}\right\}$ is a finite convex family and $B_{k}$ is a positive matrix with trace $\left(B_{k}\right)$ $=1$, if we let

$$
\begin{aligned}
K(q) & =\left\{k: \text { there is an } i \text { and } a j \text { with } \mid a_{i j}^{n}-\sum\left\{b_{t s}^{k}:(s, t)\right.\right. \\
& \left.\left.=\left(a p_{n}+i, a p_{n}+j\right), a=0, \cdots,\left(p_{q} / p_{n}\right)-1\right\} \mid>\epsilon\right\}
\end{aligned}
$$

where $B_{k}$ is the matrix $\left(b_{s t}^{k}\right)$, then

$$
\sum\left\{\alpha_{k}: k \in K(q)\right\}<\epsilon .
$$

Proof. If $A_{n} \geqq 0$ for each $n$ then by $6.1, f \mid \mathfrak{M}_{n} \geqq 0$ for each $n$. Let $D$ be a positive element of $\mathfrak{A}$ and choose $D_{n} \in \mathfrak{M}_{n}$ such that $D_{n} \rightarrow D$. Then $f(D)$ $=f\left(\left(D^{*} D\right)^{1 / 2}\right)=\lim _{n} f\left(\left(D_{n}^{*} D_{n}\right)^{1 / 2}\right) \geqq 0$ and so $f \geqq 0$. Conversely if $f \geqq 0$ then $f \mid \mathfrak{M}_{n} \geqq 0$ for each $n$ and by $6.1 A_{n} \geqq 0$ for each $n$. Also $f(I)=\sum_{i} f\left(e_{i t}^{n}\right)$ $=$ trace $\left(A_{n}\right)$ and the proof of the first statement of 2.4 is complete.

Suppose that $f$ is a state and that the inclusions $\mathfrak{M}_{n} \rightarrow \mathfrak{M}_{q}$ are standard imbeddings. Suppose that for each $n$ and $\epsilon$ we can find an $r_{3}(n, \epsilon)$ with the properties stated in 2.4. Let $r_{1}(n, \epsilon)=r_{3}\left(n, \epsilon p_{n}^{-2}\right)(=r)$, let $\phi^{-1}\left(S\left(n, \epsilon p_{n}^{-2}\right)\right)=T$. By 6.1, $T$ is an $\epsilon p_{n}^{-2}$-dense subset of $\left\{a \tau: a \in[0,1], \tau\right.$ is a state of $\mathfrak{M}_{r}$ and $\left.f \mid \mathfrak{M}_{r} \geqq a \tau\right\}$. If $D=\sum_{i j} d_{i j} e_{k j}^{n} \in \mathfrak{M}_{n}$, where $d_{i j}$ is a complex number, then $\|D\| \leqq 1$ implies $\left|d_{i j}\right| \leqq 1$ for all $i, j$, and consequently

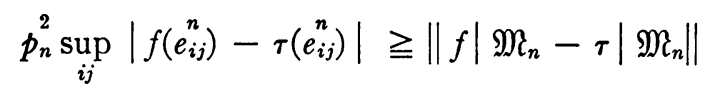

for any state $\tau$ of $\mathfrak{M}_{r}$. If $a \tau \in T$, then $a \phi(\tau) \in S\left(n, \epsilon p_{n}^{-2}\right)$ and so $\sup _{i j}\left|f\left(e_{i j}^{n}\right)-\tau\left(e_{i j}^{n}\right)\right| \leqq \epsilon p_{n}^{-2}$ or $a<\epsilon p_{n}^{-2}$. Thus $\left\|f\left|\mathfrak{M}_{n}-\tau\right| \mathfrak{M}_{n}\right\| \leqq \epsilon$ or $a<\epsilon$ and by $2.1, f$ is pure. 
Suppose that $f$ is a pure state of $\mathfrak{A}$ and let $n$ be a positive integer, let $\epsilon$ be a positive number. Let $r_{4}(n, \epsilon)=r_{2}(n, \epsilon)$. If $A_{q}=\sum_{k} \alpha_{k} B_{k}$ as in the statement of 2.4 , let $\tau_{k}=\phi^{-1}\left(B_{k}\right)$. By $6.1, \tau_{k}$ is a state of $\mathfrak{M}_{q}$ and $f \mid \mathfrak{M}_{q}=\sum_{k} \alpha_{k} \tau_{k}$. If we define $K_{q}$ as in 2.2 , then $K_{q} \supset K(q)$. Thus $\sum\left\{\alpha_{k}: k \in K(q)\right\}$ $\leqq \sum\left\{\alpha_{k}: k \in K_{q}\right\}<\epsilon$ and $r_{4}(n, \epsilon)$ has the desired properties. This completes the proof.

Lemma 2.5. Let $\mathfrak{A}$ be a UHF algebra generated by factors $\mathfrak{M}_{n}$ of type $I_{q_{n}}$. Let $\omega$ be a pure state of $\mathfrak{A}$, let $\epsilon>0$, let $n$ be a positive integer. There is an integer $r \geqq n$ and a pure state $\omega^{\prime}$ of $\mathfrak{A}$ such that

(1) $\left\|\omega^{\prime}-\omega\right\|<\epsilon$,

(2) carrier $\omega^{\prime} \mid \mathfrak{M}_{r} \leqq$ carrier $\omega \mid \mathfrak{M}_{r}$,

(3) if $\rho$ is a state of $\mathfrak{M}_{r}$ and carrier $\rho \leqq$ carrier $\omega^{\prime} \mid \mathfrak{M}_{r}$ then $\left\|\rho\left|\mathfrak{M}_{n}-\omega^{\prime}\right| \mathfrak{M}_{n}\right\|$ $<\epsilon$,

(4) $\mathfrak{M}_{r}$-dimension (carrier $\left.\omega^{\prime} \mid \mathfrak{M}_{r}\right) \leqq q_{r} / q_{n}$.

Proof. By 6.1 we have $\omega \mid \mathfrak{M}_{n}=\sum_{i=1}^{t} \alpha_{i} \tau_{i}$ where the right hand member is a finite convex sum of pure states of $\mathfrak{M}_{n}$. Let $\epsilon^{\prime}=\min \left\{\epsilon, \alpha_{1}, \cdots, \alpha_{t}\right\}$, let $\epsilon^{\prime \prime}=\min \left\{\left(\epsilon^{\prime} / 26\right)^{2}, \epsilon^{\prime} / 2 q_{n}^{2}, 1 / 2\right\}$. We let $r$ be the $r_{2}\left(\epsilon^{\prime \prime}, n\right)$ given by 2.2 . Let $F$ be the carrier of $\omega \mid \mathfrak{M}_{r}$, let $B=\left(\phi\left(\omega \mid \mathfrak{M}_{r}\right)\right)^{1 / 2}$, where $\phi$ is defined in 6.1 , let $\left\{A_{j}: j=1, \cdots, q_{n}^{2}\right\}$ be a set of matrix units for $\mathfrak{M}_{n}$. We identify $\mathfrak{M}_{r}$ and the algebra of all linear operators on $q_{r}$-dimensional Hilbert space.

By $6.1 \omega \mid \mathfrak{M}_{r}=$ trace $(B \cdot B)$, and so $F=$ range $B=I-($ null space $B$ ). We choose a maximal orthonormal family $\left\{y_{k}\right\}$ in $F$ such that for each $k$ there is a $j$ with

$$
\left|1 /\left\|B y_{k}\right\|^{2}\left(A_{j} B y_{k}, B y_{k}\right)-\omega\left(A_{j}\right)\right|>\epsilon^{\prime \prime} .
$$

Choose an orthonormal basis $\left\{z_{h}\right\}$ for $F-\left[\left\{y_{k}\right\}\right]$. By 6.1

$$
\omega \mid \mathfrak{M}_{r}=\sum_{k}\left\|B y_{k}\right\|^{2} \omega_{B y_{k} /}\left\|_{B y_{k}}\right\|+\sum_{h}\left\|B z_{h}\right\|^{2} \omega_{B z_{h} /\left\|_{B z_{h}}\right\|}
$$

By the choice of $y_{k}$,

$$
\left\|\omega_{B y_{k} /\left\|y_{k}\right\|}\left|\mathfrak{M}_{n}-\omega\right| \mathfrak{M}_{n}\right\|>\epsilon^{\prime \prime}
$$

and so by the choice of $r$,

$$
b=\sum_{k}\left\|B y_{k}\right\|^{2}<\epsilon^{\prime \prime}
$$

where $b$ is defined by the above equality. If $x \in\left[\left\{z_{h}\right\}\right]$ and $\|B x\|=1$, then by maximality of $\left\{y_{k}\right\}$,

$$
\left|\omega_{B x}\left(A_{j}\right)-\omega\left(A_{j}\right)\right| \leqq \epsilon^{\prime \prime}
$$

and

$$
\left\|\omega_{B x}\left|\mathfrak{M}_{n}-\omega\right| \mathfrak{M}_{n}\right\| \leqq q_{n}^{2} \epsilon^{\prime \prime} \leqq \epsilon^{\prime} / 2 \leqq \epsilon / 2
$$


where $\left(^{*}\right)$ follows from the fact that if $A$ is in the unit sphere of $\mathfrak{M}_{n}$, then $A=\sum_{j} \beta_{j} A_{j}$, and $\left|\beta_{j}\right| \leqq 1$.

Let $G$ be the projection (not necessarily orthogonal) onto $\left[\left\{B z_{h}\right\}\right]$ along $\left[\left\{B y_{k}\right\}\right]+I-F$, let

$$
\omega^{\prime}=(1-b)^{-1} \omega\left(G^{*} \cdot G\right) .
$$

$\omega^{\prime}$ is a positive linear functional and

$$
\begin{aligned}
\omega^{\prime}(I) & =(1-b)^{-1} \omega\left(G^{*} G\right) \\
& =(1-b)^{-1} \sum_{h}\left\|B z_{h}\right\|^{2} \\
& =(1-b)^{-1}\left(1-\sum_{k}\left\|B y_{k}\right\|^{2}\right)=1
\end{aligned}
$$

so $\omega^{\prime}$ is a state. If $\theta$ is the representation of $\mathfrak{A}$ due to $\omega$ on a Hilbert space $\mathfrak{S}_{\theta}$, then $\omega=\omega_{x} \circ \theta$ for some $x$ in $\mathfrak{S} . \omega^{\prime}=(1-b)^{-1} \omega_{\theta(G) x} \circ \theta$ is also a vector state. Since $\theta$ is irreducible, $\omega^{\prime}$ is pure. Let $x_{1}=\theta(G) x$, let $x_{2}=\theta(I-G) x$. Then

$$
\begin{aligned}
\left\|x_{2}\right\|^{2} & =\|\theta(I-G) x\|^{2} \\
& =\left(\theta\left((I-G)^{*}(I-G)\right) x, x\right) \\
& =\omega\left((I-G)^{*}(I-G)\right) \\
& =\sum_{k}\left\|B y_{k}\right\|^{2}=b
\end{aligned}
$$

and $\left\|x_{1}\right\| \leqq 1+b^{1 / 2}$. Hence if $A$ is in the unit sphere of $\mathfrak{A}$,

$$
\begin{aligned}
\left|\omega^{\prime}(A)-\omega(A)\right|= & \left|(1-b)^{-1}\left(\theta(A) x_{1}, x_{1}\right)-(\theta(A) x, x)\right| \\
\leqq & \left|b(1-b)^{-1}\left(\theta(A) x_{1}, x_{1}\right)\right|+\left|\left(\theta(A) x_{2}, x_{2}\right)\right| \\
& \left.+\left|\left(\theta(A) x_{1}, x_{2}\right)\right|+\mid(\theta(A)) x_{2}, x_{1}\right) \mid \\
\leqq & \left.b(1-b)^{-1}\left\|x_{1}\right\|\right|^{2}+\left\|x_{2}\right\|^{2}+2\left\|x_{1} \mid\right\| x_{2} \| \\
\leqq & b(1-b)^{-1}\left(1+b^{1 / 2}\right)^{2}+b+2 b^{1 / 2}\left(1+b^{1 / 2}\right) \\
\leqq & 13 b^{1 / 2}<13\left(\epsilon^{\prime \prime}\right)^{1 / 2} \leqq \epsilon / 2
\end{aligned}
$$

and $\left\|\omega^{\prime}-\omega\right\|<\epsilon / 2$ which gives us (1).

The carrier of $\omega^{\prime} \mid \mathfrak{M}_{r}$ is $\left[\left\{B z_{h}\right\}\right] \leqq$ range $B=$ carrier $\omega \mid \mathfrak{M}_{r}$ and $(2)$ is proved. Any unit vector $v$ in $\left[\left\{B z_{h}\right\}\right]$ has the form $v=B x$ for some $x$ in $\left[\left\{z_{h}\right\}\right]$. By $\left(^{*}\right)$ and the fact that $\left\|\omega^{\prime}-\omega\right\|<\epsilon / 2$ we see that (3) is true if $\rho$ is pure. Thus (3) is true in general. Let $i \in\{1, \cdots, t\}$ and let $E=$ carrier $\tau_{i}$. The mapping from carrier $\omega^{\prime} \mid \mathfrak{M}_{r}$ into $E$ given by $x \rightarrow E x$ annihilates (at least) the complement of a $q_{r} / q_{n}$ dimensional subspace of carrier $\omega^{\prime} \mid \mathfrak{M}_{r}$. If (4) does not hold we can find a unit vector $v$ in the kernel of this map. As above $v=B x$, with $x \in\left[\left\{z_{h}\right\}\right]$, and $\left|\omega_{v}(E)-\omega(E)\right| \geqq \alpha_{i} \geqq \epsilon^{\prime}$ which contradicts $(*)$. This completes the proof. 
The following lemma will be helpful in studying irreducible representations of UHF algebras.

Lemma 2.6. Let $\mathfrak{A}$ be a $U H F$ algebra generated by factors $\mathfrak{M}_{n}$, let $\omega$ be a pure state of $\mathfrak{A}$. There is a subsequence $\left\{n_{i}\right\}$ of the positive integers and a sequence $\left\{E_{i}\right\}$ of projections, $E_{i} \in \mathfrak{M}_{n_{i}}$, such that

(a) $1-\omega\left(E_{i}\right)<2^{-i}$,

(b) if $\rho$ is a state of $\mathfrak{M}_{n_{i+1}}$ and carrier $\rho \leqq E_{i+1}$ then $\left\|\rho\left|\mathfrak{M}_{n_{i}}-\omega\right| \mathfrak{M}_{n_{i}}\right\|<2^{-i}$ for $i=1,2, \cdots$.

Proof. Let $E_{1}=I, n_{1}=1$. Suppose $n_{j}$ and $E_{j}$ have been chosen for some positive integer $j$. For $n=n_{j}$ and for $\epsilon=2^{-j-2}$ find an $r$ and an $\omega^{\prime}$ which satisfy 2.5. Let $n_{j+1}=r$, let $E_{j+1}=$ carrier $\omega^{\prime} \mid \mathfrak{M}_{n_{j+1}}$. Then

$$
\begin{aligned}
1-\omega\left(E_{j+1}\right) & =\omega^{\prime}\left(E_{j+1}\right)-\omega\left(E_{j+1}\right) \\
& \leqq\left\|\omega^{\prime}-\omega\right\|<2^{-j-1} .
\end{aligned}
$$

If $\rho$ satisfies the hypotheses of (b) then

$$
\begin{aligned}
\left\|\rho\left|\mathfrak{M}_{n_{j}}-\omega\right| \mathfrak{M}_{n_{j}}\right\| & \leqq\left\|\rho\left|\mathfrak{M}_{n_{j}}-\omega^{\prime}\right| \mathfrak{M}_{n_{j}}\right\|+\left\|\omega^{\prime}-\omega\right\| \\
& <2^{-j-2}+2^{-j-2}=2^{-j-1}
\end{aligned}
$$

and the proof of 2.6 is complete.

The next result gives another set of conditions which are necessary and sufficient for a state $\omega$ of a UHF algebra $\mathfrak{A}$ to be pure. In fact if $\omega$ is a state of $\mathfrak{A}$ and if there exist factors $\mathfrak{N}_{i}, i=1,2, \cdots$, which generate $\mathfrak{A}$ so that 2.7 formula (3) holds, then $\omega$ is pure. We remark that in the terminology of the following theorem, the sequence of projections $\left\{\right.$ carrier $\left.\omega \mid \mathfrak{N}_{i}\right\}$, the state $\omega$, and the factors $\mathfrak{N}_{i}$ satisfy 2.6 and also the sequence $\left\{\right.$ carrier $\left.\omega \mid \mathfrak{N}_{i}\right\}$ is monotone decreasing.

THEOREM 2.7. Let $\mathfrak{A}$ be a UHF algebra generated by factors $\mathfrak{M}_{n}$ of type $I_{q_{n}}$, let $\omega$ be a pure state of $\mathfrak{A}$. There is an ascending sequence $\left\{\mathfrak{N}_{i}\right\}$ of factors which generate $\mathfrak{A}$ and for each $\epsilon>0$ there is a pure state $\omega^{\prime}$ of $\mathfrak{A}$ and a subsequence $\left\{n_{i}\right\}$ of the positive integers such that

(1) $\left\|\omega-\omega^{\prime}\right\|<\epsilon$

(2) (resp. (3)) if $\rho$ is a state of $\mathfrak{M}_{n_{i}}$ (resp. $\mathfrak{N}_{i}$ ) and carrier $\rho \leqq$ carrier $\omega^{\prime} \mid \mathfrak{M}_{n_{i}}$ (resp. $\left.\omega \mid \mathfrak{N}_{i}\right)$ then $\left\|\rho\left|\mathfrak{M}_{n_{i-1}}-\omega^{\prime}\right| \mathfrak{M}_{n_{i-1}}\right\|\left(\right.$ resp. $\left.\left\|\rho\left|\mathfrak{N}_{i-1}-\omega\right| \mathfrak{N}_{i-1}\right\|\right)<2^{-i}$,

(4) $\mathfrak{M}_{n_{i}}$ dimension carrier $\omega^{\prime} \mid \mathfrak{M}_{n_{i}} \leqq q_{n_{i}} / q_{n_{i-1}}$,

(5) $\mathfrak{N}_{i}$-dimension carrier $\omega \mid \mathfrak{N}_{i} \leqq q_{n_{i}} / q_{n_{i-1}}$.

Proof. We suppose $\epsilon<1$. Let $\omega_{1}=\omega$ and $n_{1}=1$. By induction, using 2.5, we can find a sequence $\left\{\omega_{i}\right\}$ of pure states and a subsequence $\left\{n_{i}\right\}$ of the positive integers such that $\left\|\omega_{i-1}-\omega_{i}\right\|<\epsilon / 2^{i+1}$, such that if $E_{i j}=$ carrier $\omega_{i} \mid \mathfrak{M}_{n_{j}}$ then $E_{i i} \leqq E_{i-1 i}$ and $\mathfrak{M}_{n_{i}}$-dimension $E_{i i} \leqq q_{n_{i}} / q_{n_{i-1}}$ and such that if $\rho$ is a state of $\mathfrak{M}_{n_{i}}$ and if carrier $\rho \leqq E_{i i}$ then $\| \rho\left|\mathfrak{M}_{n_{i-1}}-\omega_{i}\right| \mathfrak{M}_{n_{i-1}} \mid<\epsilon / 2^{i+1}$ for 
$i=2,3, \cdots\left\{\omega_{i}\right\}$ is a Cauchy sequence (in norm) in $\mathfrak{P}^{*}$, the dual to the vector space $\mathfrak{A}$, and so $\left\{\omega_{i}\right\}$ has a limit $\omega^{\prime}$ in $\mathfrak{A}^{*}, \omega^{\prime}$ is a state, and $\left\|\omega-\omega^{\prime}\right\|<\epsilon / 2$.

If $(j, k)$ is a pair of integers with $j \geqq k \geqq 1$ then $E_{j k} \leqq E_{k k}$. In fact this is true for $j=k$, suppose it is true for pairs $(s, t)$ of positive integers with $0 \leqq s-t<j-k$. Then $E_{j k+1} \leqq E_{k+1 k+1} \leqq E_{k k+1}$ and so $E_{j k} \leqq E_{k k}$. Let $E_{i}=$ carrier $\omega^{\prime} \mid \mathfrak{M}_{n_{j}}$. For any positive integer $k, \omega^{\prime}\left(I-E_{k k}\right)=\lim _{j} \omega_{j}\left(I-E_{k k}\right)=0$. Thus $E_{k}$ $\leqq E_{k k}$ and (4) is proved. If $\rho$ satisfies the hypotheses of (2), then

$$
\begin{aligned}
\| \rho\left|\mathfrak{M}_{n_{i-1}}-\omega^{\prime}\right| \mathfrak{M}_{n_{i-1}} \mid & \leqq\left\|\rho\left|\mathfrak{M}_{n_{i-1}}-\omega_{i}\right| \mathfrak{M}_{n_{i-1}}\right\|+\left\|\omega_{i}-\omega^{\prime}\right\| \\
& <\epsilon / 2^{2^{i+1}}+\epsilon / 2^{i+1}<2^{-i}
\end{aligned}
$$

and (2) is proved.

We show that $\omega^{\prime}$ is pure. Let $\mathfrak{A}_{0}$ be the $C^{*}$-algebra generated by $I$ and $\left\{E_{i}: i=1, \cdots\right\} . \mathfrak{A}_{0}$ is commutative and $\omega^{\prime}$ is a homomorphism of $\mathfrak{A}_{0}$ so $\omega^{\prime} \mid \mathfrak{A}_{0}$ is pure. If $\tau$ is any extension of $\omega^{\prime} \mid \mathfrak{A}_{0}$ to a state of $\mathfrak{A}$ then carrier $\tau \mid \mathfrak{M}_{n_{i}}$ $\leqq E_{i}$ and by (2), $\tau=\omega^{\prime}$. Thus $\omega^{\prime} \mid \mathfrak{A}_{0}$ has a unique state extension to $\mathfrak{A}$ and so $\omega^{\prime}$ is pure. A proof that $\omega^{\prime}$ is pure could also be based upon 3.2. By [1] there is a ${ }^{*}$-automorphism $\theta$ of $\mathfrak{A}$ such that $\omega^{\prime}\left(\theta^{-1}(A)\right)=\omega(A)$ for all $A \in \mathfrak{A}$. $(\theta$ is the inner automorphism from some unitary $U \in \mathfrak{R})$. Let $\mathfrak{R}_{i}=\theta\left(\mathfrak{M}_{n_{\mathfrak{i}}}\right)$. Then $\left\{\mathfrak{R}_{i}\right\}$ satisfies (3) and (5) and the proof is complete.

THEOREM 2.8. If $\mathfrak{A}$ is a UHF algebra, then the $w^{*}$ closure of the set of pure states of $\mathfrak{A}$ is the state space of $\mathfrak{A}$.

Proof. Let $\omega$ be a state of $\mathfrak{A}$, let $\mathfrak{A}$ be generated by factors $\mathfrak{M}_{n}$. Let $A_{i} \in \mathfrak{A}, i=1, \cdots, r$. We show that there is a pure state $\tau$ such that $\left|\tau\left(A_{i}\right)-\omega\left(A_{i}\right)\right|<1$ for $i=1, \cdots, r$. There is an integer $n$ and $B_{i} \in \mathfrak{M}_{n}$ with $\left\|B_{i}-A_{i}\right\|<1 / 2, i=1, \cdots, r$. If $\mathfrak{M}_{n}$ is of type $I_{r}$ we choose an integer $m$ such that $\mathfrak{M}_{m}$ is of type $I_{s}$ with $s \geqq r^{2}$. We assert that $\omega \mid \mathfrak{M}_{n}$ has an extension to a pure state $\tau^{\prime}$ of $\mathfrak{M}_{m}$. Let $\mathfrak{M}_{m}$ be represented on complex $s$-space. In this representation, the commutant $\mathfrak{M}_{n}^{\prime}$ of $\mathfrak{M}_{n}$ is a factor of type $I_{s / r}$, and $s / r \geqq r$. Thus $\mathfrak{M}_{n}$ has a separating vector, and all normal states (that is all states) of $\mathfrak{M}_{n}$ are vector states, and so they are restrictions to $\mathfrak{M}_{n}$ of pure states of $\mathfrak{M}_{m}$. This gives us the desired pure state $\tau^{\prime}$ of $\mathfrak{M}_{m}$. Let $\tau$ be a pure extension of $\tau^{\prime}$ to $\mathfrak{A}$. Then

$$
\begin{aligned}
\left|\tau\left(A_{i}\right)-\omega\left(A_{i}\right)\right| & \leqq\left|\tau\left(A_{i}\right)-\tau\left(B_{i}\right)\right|+\left|\tau\left(B_{i}\right)-\omega\left(B_{i}\right)\right|+\left|\omega\left(B_{i}\right)-\omega\left(A_{i}\right)\right| \\
& <1 / 2+0+1 / 2=1
\end{aligned}
$$

and the proof is complete.

Let $\rho$ and $\tau$ be two distinct pure states of $\mathfrak{A}$ (such pure states exist). Then $\rho / 2+\tau / 2$ is a state of $\mathfrak{A}$ which is not pure, so the set of pure states of $\mathfrak{A}$ is not $w^{*}$-closed.

A plausible noncommutative extension of the Stone-Weierstrass approximation theorem states: If $\mathfrak{A}$ and $\mathfrak{B}$ are $C^{*}$-algebras with $\mathfrak{A} \subseteq \mathfrak{B}$ and if $\mathfrak{A}$ 
separates the pure states of $\mathfrak{B}$, then $\mathfrak{A}=\mathfrak{B}$. In course lectures (1957-1958), $\mathrm{R}$. Kadison conjectured that the correct result should assume: $\mathfrak{A}$ separates the $w^{*}$-closure of the pure states of $\mathfrak{B}$ (i.e. the pure state space of $\mathfrak{B}$ ). It is automatic that the pure states are $w^{*}$-closed in the commutative (classical) case. It is known that if $\mathfrak{A}$ separates the states of $\mathfrak{B}$, then $\mathfrak{A}=\mathfrak{B}$. Thus 2.8 establishes the emended conjecture for UHF algebras. We shall show elsewhere that this emended conjecture is valid for those $C^{*}$-algebras which have a faithful irreducible representation.

3. Irreducible representations. In this section we determine the distinct classes of unitarily equivalent irreducible representations of a UHF algebra $\mathfrak{A}$. Since each irreducible representation of $\mathfrak{A}$ is unitarily equivalent to the representation due to some pure state of $\mathfrak{A}$ and since we have already determined the set of pure states of $\mathfrak{A}$, we have still to determine which pure states of $\mathfrak{A}$ give rise to unitarily equivalent representations (3.4). In 3.5 we prove that all irreducible representations of UHF algebras are on separable Hilbert space.

Lemma 3.1. If $\mathfrak{A}$ is a UHF algebra generated by factors $\mathfrak{M}_{n}$, then the unitary group of $\mathfrak{A}$ is the uniform closure of the unitary group of $\cup_{\mathfrak{M}_{n}}$.

Proof. An element $U$ of $\mathfrak{A}$ is unitary if and only if $U U^{*}=U^{*} U=I$. Multiplication and ${ }^{*}$ are continuous, so the closure of the unitary group of $\cup \mathfrak{M}_{n}$ is in the unitary group of $\mathfrak{A}$. Let $U$ be a unitary operator in $\mathfrak{A}$, let $A_{n} \in \mathfrak{M}_{n}$ be such that $A_{n} \rightarrow U$. For sufficiently large $n, A_{n}$ is invertible, and $\left(A_{n}{ }^{*} A_{n}\right)^{-1 / 2} \rightarrow\left(U^{*} U\right)^{-1 / 2}=I$. Thus $A_{n}\left(A_{n}{ }^{*} A_{n}\right)^{-1 / 2} \rightarrow U$. Since $A_{n}\left(A_{n}{ }^{*} A_{n}\right)^{-1 / 2}$ is unitary, the proof is complete.

We determine (3.2 and 3.3) the topology on the pure states of a UHF algebra $\mathfrak{A}$ which is induced by the norm in the dual to the Banach space $\mathfrak{A}$. This topology is closely related to the Hilbert spaces upon which $\mathfrak{A}$ is irreducibly represented (cf. also the remark following 3.4).

Let $\mathfrak{A}$ be a $C^{*}$-algebra, let $\left\{\rho_{\beta}: \beta \in B\right\}$ be a family of pure states of $\mathfrak{A}$ which contains exactly one representative from each unitary equivalence class of pure states of $\mathfrak{A}$. Let $\phi$ be the representation of $\mathfrak{A}$ on the Hilbert space $\mathfrak{S}$ which is the direct sum of the representations $\phi_{\beta}$ on Hilbert spaces $\mathfrak{S}_{\beta}$ due to $\rho_{\beta}, \beta \in B$. We identify $\mathfrak{A}$ and $\phi(\mathfrak{A})$. ( $\phi$ is faithful.)

Lemma 3.2. Let $\mathfrak{A}$ be a $C^{*}$-algebra acting on the Hilbert space $\mathfrak{S}$ as above. Let $x, y \in \mathfrak{S}$ be such that $\omega_{x} \mid \mathfrak{A}$ and $\omega_{y} \mid \mathfrak{A}$ are pure states of $\mathfrak{A}$. Then

$$
\begin{aligned}
1-|(x, y)|^{2} & \leqq\left\|\omega_{x}\left|\mathfrak{X}-\omega_{y}\right| \mathfrak{x}\right\| \\
& \leqq \inf \{2\|\alpha x-y\|: \alpha \text { is a complex number, }|\alpha|=1\} \\
& \leqq 2^{3 / 2}\left(1-|(x, y)|^{2}\right)^{1 / 2} .
\end{aligned}
$$

Proof. By $[1][x] \in \mathfrak{A}-$, the strong closure of $\mathfrak{A}$, and 


$$
\begin{aligned}
1-|(x, y)|^{2} & =1-((y, x) x, y) \\
& =1-([x] y, y) \\
& \leqq\left\|\omega_{x}\left|\mathfrak{A}--\omega_{y}\right| \mathfrak{A}-\right\| \\
& =\left\|\omega_{x}\left|\mathfrak{A}-\omega_{y}\right| \mathfrak{A}\right\|
\end{aligned}
$$

where the last equality follows from [3].

Let $A$ be in the unit sphere of $\mathfrak{A}$, let $\alpha$ be a complex number of modulus 1 . Then (for any vectors $x, y$ in the unit sphere of $\mathfrak{S}$ )

$$
\begin{aligned}
|(A x, x)-(A y, y)| & \leqq|(A \alpha x, \alpha x)-(A \alpha x, y)|+|(A \alpha x, y)-(A y, y)| \\
& \leqq 2\|\alpha x-y\| .
\end{aligned}
$$

For some complex $\alpha$ of modulus $1,(\alpha x, y) \geqq 0$ and

$$
\begin{aligned}
\|\alpha x-y\|^{2} & =(\alpha x, \alpha x)-(\alpha x, y)-(y, \alpha x)+(y, y) \\
& =2(1-|(x, y)|) \leqq 2\left(1-|(x, y)|^{2}\right)
\end{aligned}
$$

and the proof is complete.

Lemma 3.3. Let $\mathfrak{A}$ be a UHF algebra acting as preceding 3.2 on the Hilbert space $\mathfrak{S}$, let $\mathfrak{A}$ be generated by factors $\mathfrak{M}_{n}$, let $\omega=\omega_{x} \mid \mathfrak{A}$ be a pure state of $\mathfrak{A}$ for some $x$ in $\mathfrak{S}$. If $\left\{E_{i}\right\}$ is the sequence of projections determined by 2.6 for the pure state $\omega$ then strong $\lim E_{i}=[x]$ and $|(x, y)|^{2}=\lim \omega_{y}\left(E_{i}\right)$ for any $y$ in $\mathfrak{S}$.

Proof. Let $\left\{n_{i}\right\}$ be the subsequence of the positive integers determined by 2.6. We assert that there is a sequence $\{\epsilon(i)\}$ of positive numbers such that $\epsilon(i) \rightarrow 0$ and $E_{i}+\epsilon(i) I>E_{s}$ if $s>i$. If $s>i$,

$$
\begin{aligned}
\left\|E_{i} E_{s} E_{i}-E_{s}\right\| & \leqq\left\|E_{i} E_{s} E_{i}-E_{i} E_{s}\right\|+\left\|E_{i} E_{s}-E_{8}\right\| \\
& \leqq 2\left\|E_{i} E_{s}-E_{s}\right\|
\end{aligned}
$$

and if $z$ is a unit vector in $E_{8}$,

$$
\begin{aligned}
\left\|E_{i} z-z\right\|^{2} & =\left(E_{i} z, E_{i} z\right)+(z, z)-\left(E_{i} z, z\right)-\left(z, E_{i} z\right) \\
& =1-\left(E_{i} z, z\right) \\
& <1-\omega\left(E_{i}\right)+2^{-s+1}<2^{-i+1}
\end{aligned}
$$

since $\left\|\omega_{z}\left|\mathfrak{M}_{n_{s-1}}-\omega\right| \mathfrak{M}_{n_{s-1}}\right\|<2^{-s+1}$ and $1-\omega\left(E_{i}\right)<2^{-i}$. Thus $\left\|E_{i} E_{8} E_{i}-E_{s}\right\|$ $<2^{(-i+3) / 2}$. Let $\epsilon(i)=2^{(-i+3) / 2}$. Since $I \geqq E_{8}, E_{i} \geqq E_{i} E_{8} E_{i}$, and

$$
E_{i}+\epsilon(i) I \geqq E_{i} E_{8} E_{i}+\epsilon(i) I \geqq E_{i} E_{8} E_{i}+E_{8}-E_{i} E_{8} E_{i}=E_{8},
$$

which proves the assertion.

For the time being we do not assume $\mathfrak{S}$ and the action of $\mathfrak{A}$ on $\mathfrak{S}$ is as preceding 3.2, we do not assume that $\omega$ is a vector state. Let

$$
F=\left[\left\{z: z \in \mathfrak{D}, \omega_{z} \mid \mathfrak{A}=\omega\right\}\right],
$$


we assert that strong $\lim E_{i}=F$. Let $\{i(j)\}$ be a subsequence of the positive integers. We show that there is a subsequence $\{i(j(k))\}$ of $\{i(j)\}$ such that $E_{i(j(k))}+2 \epsilon(i(j(k))) I>E_{i(j(k+1))}+2 \epsilon(i(j(k+1))) I$. Let $j(1)=1$, and suppose $j(k)$ has been chosen for some positive integer $k$. Choose $j(k+1)$ so that $\epsilon(i(j(k))) \geqq 2 \epsilon(i(j(k+1)))$. Then $\{i(j(k))\}$ has the above property. Thus by monotone convergence every subsequence of $\left\{E_{i}\right\}$ has a strongly convergent subsequence. Let $E$ be the limit of a strongly convergent subsequence $\left\{E_{i(j)}\right\}$. We show $E=F$ which will prove our assertion. $E^{2}=$ strong $\lim E_{i(j)}^{2}$ $=E$ and $E^{*}=$ weak $\lim E_{\mathfrak{i}(j)}^{*}=E$ so $E$ is a projection. Suppose that $z \in \mathfrak{S}$ is such that $\omega_{z} \mid \mathfrak{A}=\omega$. As in (1), $\left\|E_{i} z-z\right\|^{2}=1-\left(E_{i} z, z\right)<2^{-i}$ so $z \in E$. Suppose that $y$ is a unit vector in $E$. Then

$$
\begin{aligned}
\left\|\omega_{y}\left|\mathfrak{M}_{n_{i-1}}-\omega\right| \mathfrak{M}_{n_{i-1}}\right\| \leqq & \left\|\omega_{y}-\omega_{E_{i} y}\right\|+\left\|\omega_{E_{i y}}-1 /\right\| E_{i} y\left\|^{2} \omega_{E_{i} y}\right\| \\
& +\|1 /\| E_{i} y\left\|^{2} \omega_{E_{i \nu}}\left|\mathfrak{M}_{n_{i-1}}-\omega\right| \mathfrak{M}_{n_{i-1}}\right\| \\
\leqq & 2\left\|E_{i} y-y\right\|+1-\left\|E_{i} y\right\|^{2}+2^{-i+1}
\end{aligned}
$$

where the first term in the last inequality is due to the second paragraph of the proof of 3.2 , and the last term is due to carrier $1 /\left.\left\|E_{i} y\right\|\right|^{2} \omega_{E_{i} y} \mid \mathfrak{M}_{n_{i}} \leqq E_{i}$. Thus $\omega_{y} \mid \mathfrak{A}=\omega, y \in F$, and strong $\lim E_{i}=F$. If $\mathfrak{A}$ and $\mathfrak{T}$ are as preceding Lemma 3.2 then $[F]=[x]$ by Lemma 3.2 and the first statement of Lemma 3.3 is proved.

If $y \in \mathfrak{S}$, then $\lim \left(E_{i} y, y\right)=([x] y, y)=|(x, y)|^{2}$ and the proof of 3.3 is complete.

THEOREM 3.4. Let $\mathfrak{A}$ be a UHF algebra generated by factors $\mathfrak{M}_{n}$, let $\rho$ and $\tau$ be pure states of $\mathfrak{A}$, let $\left\{E_{i}\right\}$ be a sequence of projections chosen by 2.6 for the pure state $\tau$. Suppose that $0 \leqq r<1 / 2$ and that for each positive integer $n$ we are given a subset $\mathfrak{U}_{n}$ of the unitary group of $\mathfrak{M}_{n}$ such that if $W$ is a unitary operator in $\mathfrak{M}_{n}$ there is a $V$ in $\mathfrak{U}_{n}$ with $\|V-W\| \leqq r$. The following statements are equivalent.

(1) The representations $\phi_{\rho}$ and $\phi_{\tau}$ due to $\rho$ and $\tau$ respectively are unitarily equivalent.

(2) There is a unitary $U$ in $\mathfrak{A}$ with $\rho\left(U^{*} \cdot U\right)=\tau$.

(3) There is a positive integer $k$ such that $\lim \sup \left\{\sup \left\{\rho\left(V^{*} E_{i} V\right): V \in \mathfrak{U}_{k}\right\}: i=1, \cdots\right\}>0$.

(4) There is a positive integer $k$ such that

$$
\lim _{i}\left(\inf \left\{\text { trace }\left|\phi\left(\left(\rho\left(V^{*} \cdot V\right)-\tau\right) \mid M_{i}\right)\right|: V \in \mathfrak{U}_{k}\right\}\right)<1,
$$

where $\phi$ is defined by 6.1 .

Proof. We assert that if $f$ and $g$ are positive linear functionals on $\mathfrak{M}_{n}$ then $\|f-g\|=$ trace $|\phi(f-g)|$. Let $f_{0}$ (resp. $g_{0}$ ) be the linear functional on $\mathfrak{M}_{n}$ such that $\phi\left(f_{0}\right)$ (resp. $-\phi\left(g_{0}\right)$ ) is the positive (resp. negative) part of the selfadjoint operator $\phi(f-g)$. Then $f_{0}, g_{0} \geqq 0$ and $f_{0}-g_{0}=f-g$. Carrier $f_{0}$ (resp. $g_{0}$ ) 
$=$ range $\phi\left(f_{0}\right)$ (resp. $\left.\phi\left(g_{0}\right)\right)$, in fact by 6.1 this is true for $f_{0}$ a pure state, thus it is true for $f_{0} \geqq 0$. Thus carrier $f_{0} \perp$ carrier $g_{0}$ and

$$
\begin{aligned}
\|f-g\| & =\left\|f_{0}-g_{0}\right\|=\left\|f_{0}\right\|+\left\|g_{0}\right\|=f_{0}(I)+g_{0}(I) \\
& =\operatorname{trace} \phi\left(f_{0}\right)+\operatorname{trace} \phi\left(g_{0}\right)=\operatorname{trace} \phi\left(f_{0}+g_{0}\right) \\
& =\operatorname{trace}\left|\phi\left(f_{0}-g_{0}\right)\right|=\operatorname{trace}|\phi(f-g)|
\end{aligned}
$$

as asserted.

The implications (1) $\Leftrightarrow(2)$ follow from [1]. Suppose (2) is satisfied. Let $\mathfrak{A}$ be acting on $\mathfrak{S}$ as preceding 3.2. There are $x, y \in \mathfrak{S}$ with $\omega_{x}\left|\mathfrak{A}=\tau, \omega_{y}\right| \mathfrak{A}=\rho$, and a unitary $U \in \mathfrak{A}$ with $\omega_{U y} \mid \mathfrak{A}=\tau$. By $3.2 \gamma U y=x$ where $\gamma$ is some complex number of modulus 1 . There is a positive integer $k$ and a unitary $W \in \mathfrak{M}_{k}$ with $\|W-U\|<(1-2 r) / 4$, there is a $V \in \mathfrak{U}_{k}$ with $\|V-W\| \leqq r$. For any positive integer $i$,

$$
\begin{aligned}
\operatorname{trace}\left|\phi\left(\left(\rho\left(V^{*} \cdot V\right)-\tau\right) \mid \mathfrak{M}_{i}\right)\right| & =\left\|\left(\rho\left(V^{*} \cdot V\right)-\tau\right) \mid \mathfrak{M}_{i}\right\| \\
& \leqq\left\|\omega_{V \nu} \mid \mathfrak{A}-\tau\right\| \leqq 2\|\gamma V y-x\| \\
& \leqq 2\|V-U\| \leqq 2\|V-W\|+2\|W-U\| \\
& <2 r-(1-2 r) / 2=1 / 2+r<1 .
\end{aligned}
$$

Thus trace $\left|\phi\left(\left(\rho\left(V^{*} \cdot V\right)-\tau\right) \mid \mathfrak{M}_{i}\right)\right|$ is monotone increasing (as $i$ increases), the limit in (4) exists and is less than 1 , and (4) is satisfied.

Suppose (4) holds. Let $\left\{n_{i}\right\}$ be the subsequence of the positive integers determined by 2.6 . in conjunction with $\left\{E_{i}\right\}$. Then for some positive integer $k$ and some number $\delta$, with $0 \leqq \delta<1$, we can choose $V(i) \in \mathfrak{U}_{k}$ such that trace $\left|\phi\left(\left(\rho\left(V(i)^{*} \cdot V(i)\right)-\tau\right) \mid \mathfrak{M}_{n_{\mathfrak{i}}}\right)\right|<\delta$, and

$$
\begin{aligned}
\rho\left(V(i)^{*} E_{i} V(i)\right) & \geqq \tau\left(E_{i}\right)-\left\|\left(\rho\left(V(i)^{*} \cdot V(i)\right)-\tau\right) \mid \mathfrak{M}_{n_{i}}\right\| \\
& \geqq 1+2^{-i}-\delta .
\end{aligned}
$$

For large $i, \rho\left(V(i)^{*} E_{i} V(i)\right)>0$ and (3) is satisfied. If (3) is true there is an integer $k$, a cofinal subset $J$ of the positive integers and an $\epsilon>0$ such that for each $j \in J$ there is a $V(j) \in \mathfrak{U}_{k}$ with $\rho\left(V(j) * E_{j} V(j)\right)>\epsilon$. Since the unitary group of $\mathfrak{M}_{k}$ is compact, we can choose a unitary $V \in \mathfrak{M}_{k}$ which is a limit point of $\{V(j): j \in J\}$. We have $\rho\left(V^{*} E_{j} V\right) \geqq \epsilon / 2$ for all $j$ in some cofinal subset of $J$ and by 3.3, $|(x, V y)|^{2} \geqq \epsilon / 2$. Recall that $\mathfrak{S}=\sum \oplus\left\{\mathfrak{S}_{\beta}: \beta \in B\right\}$, so $V y$ $=\sum\left\{z_{\beta}: \beta \in B\right\}$, with $z_{\beta} \in \mathfrak{S}_{\beta}$, and $\omega_{V y} \mid \mathfrak{A}=\sum \omega_{\boldsymbol{z}_{\beta}}$. However $\omega_{V y} \mid \mathfrak{A}$ is pure since $\omega_{y} \mid \mathfrak{A}$ is pure and so there is a unique $\beta_{0} \in B$ with $z_{\beta_{0}} \neq 0$. By the same argument $x \in \mathfrak{S}_{\beta_{1}}$ for some $\beta_{1} \in B$. Since $(x, V y) \neq 0$, we have $\beta_{0}=\beta_{1}$. Recalling that $A \rightarrow A \mathfrak{S}_{\beta_{1}}$ is an irreducible representation of $\mathfrak{A}$, the representation due to $\omega_{x} \mid \mathfrak{A}$ is unitarily equivalent to the representation due to $\omega_{V y} \mid \mathfrak{A}$ which is unitarily equivalent to the representation due to $\omega_{y} \mid \mathfrak{A}$. This completes the proof of 3.4. 
We remark that if $f$ and $g$ are positive linear functionals on a UHF algebra $\mathfrak{A}$ which is generated by factors $\mathfrak{M}_{n}$ then by the first paragraph of the proof of 3.4 ,

$$
\|f-g\|=\lim \left\|(f-g) \mid \mathfrak{M}_{n}\right\|=\lim \text { trace }\left|\phi\left((f-g) \mid \mathfrak{M}_{n}\right)\right| .
$$

THEOREM 3.5. Each irreducible representation of a UHF algebra is on separable Hilbert space.

Proof. Let $\phi$ be an irreducible representation of a UHF algebra $\mathfrak{A}$ on a Hilbert space $\mathfrak{H}$, let $\mathfrak{A}$ be generated by factors $\mathfrak{M}_{n}$. If $x$ is a nonzero vector in $\mathfrak{S}$, then $\bigcup_{n}\left[\phi\left(\mathfrak{M}_{n}\right) x\right]$ is separable and is dense in $\mathfrak{S}$.

\section{Representations of type $\mathrm{II}_{1}$.}

THEOREM 4.1. Let $\mathfrak{A}$ be a UHF algebra and let $\phi$ be a representation of $\mathfrak{A}$ such that $\phi(A)^{-}$, the weak closure of $\phi(\mathfrak{H})$, is a finite von Neumann algebra. Then $\phi(\mathfrak{A})^{-}$is a factor (and is hyperfinite).

Proof. Let $及$ be the center of $\phi(\mathfrak{A})^{-}$. Suppose $E$ and $F$ are two orthogonal nonzero projections in $\mathbb{3}$. There are finite normal traces $\theta$ and $\psi$ of $\phi(\mathfrak{A})^{-}$ such that $\theta(E) \neq 0$ and $\psi(F) \neq 0$. Let $\theta^{\prime}(A)=\theta(E A)$ and $\psi^{\prime}(A)=\psi(F A)$ for $A \in \phi(\mathfrak{H})^{-}$. Then $\theta^{\prime}$ and $\psi^{\prime}$ are normal finite traces of $\phi(A)^{-}$. However $\lambda \theta^{\prime}\left|\cup \phi\left(\mathfrak{M}_{n}\right)=\psi^{\prime}\right| \cup \psi\left(\mathfrak{M}_{n}\right)$ for some $\lambda>0$ where $\mathfrak{A}$ is generated by factors $\mathfrak{M}_{n}$. $\theta^{\prime}$ and $\psi^{\prime}$ are ultra-weakly continuous, so $\lambda \theta^{\prime}=\psi^{\prime}$. Thus $0 \neq \lambda \theta^{\prime}(E)=\psi^{\prime}(E)=0$, a contradiction, and there do not exist two orthogonal nonzero projections in 3 , which implies that $\phi(\mathfrak{A})^{-}$is a factor.

5. An example. We construct a $C^{*}$-algebra $\mathfrak{A}_{0}$ which satisfies:

(1) $\mathfrak{A}_{0}$ is not a UHF algebra.

(2) The center of $\mathfrak{H}_{0}$ consists of scalar multiples of the identity.

(3) There is a strictly ascending sequence $\left\{\mathfrak{N}_{i}: i=1,2, \ldots\right\}$ of finite dimensional von Neumann algebras in $\mathfrak{A}_{0}$ and $\bigcup_{i} \mathfrak{N}_{i}$ is dense in $\mathfrak{A}_{0}$.

This example is related to one of the definitions of hyperfinite factors of type $\mathrm{II}_{1}[4]$. We will show that $\mathfrak{A}_{0}$ is not a UHF algebra by showing that $\mathfrak{A}_{0}$ does not satisfy the conclusion of the following theorem.

Theorem 5.1. If $\mathfrak{A}$ is a UHF algebra, then $\mathfrak{A}$ is simple.

Proof. Let $\mathfrak{A}$ be generated by factors $\mathfrak{M}_{i}$. Let $\Im$ be a proper two sided ideal in $\mathfrak{A}$. $\Im$ is contained in a maximal two sided proper ideal $\Re$ which is necessarily closed. The natural mapping $\phi: \mathfrak{A} \rightarrow \mathfrak{A} / \mathfrak{\Omega}$ is a *-homomorphism. Since $\Re$ is proper and $\mathfrak{M}_{i}$ is simple, $\phi \mid \mathfrak{M}_{i}$ is a ${ }^{*}$-isomorphism and hence is isometric. Thus $\phi$ is isometric and so $\Re=\{0\}, \Im=\{0\}$ and the proof is complete.

Let $\mathfrak{A}$ be a UHF algebra generated by factors $\mathfrak{M}_{i}, \mathfrak{M}_{i} \neq \mathfrak{M}_{i+1}$. Let $\rho$ be a state of $\mathfrak{A}$ such that $\rho \mid \mathfrak{M}_{i}$ is pure for $i=1,2, \ldots$. Such states exist, and by 2.1 are pure. Let $\phi$ be the representation of $\mathfrak{A}$ due to $\rho$ on a Hilbert space $\mathfrak{S}$. We identify $\mathfrak{A}$ and $\phi(\mathfrak{A})$. Let $x$ be a unit vector of $\mathfrak{S}$ such that $\rho=\omega_{x} \mid \mathfrak{A}$. Let 


$$
\mathfrak{A}_{0}=\{A: A \in \mathfrak{A},[x] A=A[x]\} .
$$

$\mathfrak{H}_{0}$ is a $C^{*}$-algebra, we assert that $\mathfrak{A}_{0}$ satisfies (1), (2), and (3). $\mathfrak{A}_{0}$ is not a UHF algebra since

$$
\Im=\left\{A: A \in \mathfrak{A}_{0}, A x=0\right\}
$$

is a proper two sided ideal in $\mathfrak{A}_{0}$, and by [2] $\Im \neq\{0\}$. To show that $\mathfrak{A}_{0}$ satisfies (2), we prove the stronger statement that $\mathfrak{A}_{0}$ has a faithful irreducible representation. In fact the mapping $\Pi: A \rightarrow A(I-[x])$ is a representation of $\mathfrak{A}_{0}$ on the Hilbert space $I-[x]$ and by $[2], \Pi\left(\mathfrak{A}_{0}\right)$ acts irreducibly on $I-[x]$. If $A$ is in the kernel of $\Pi$, then $[x] A=A[x]=A$, so $A$ is a completely continuous operator. Let $\mathbb{E}$ be the set of all completely continuous operators on $\mathfrak{S}$. Then $\mathfrak{S} \cap \mathfrak{A}$ is a proper two sided ideal in $\mathfrak{A}$ and by $5.1, \mathfrak{C} \cap \mathfrak{A}=\{0\}$. Thus $\mathfrak{E} \cap \mathfrak{A}_{0}=\{0\}, \Pi$ is faithful and $\mathfrak{A}_{0}$ satisfies (2). Let $E_{i}=$ carrier $\rho \mid \mathfrak{M}_{i}$. $E_{i}$ is a minimal projection in $\mathfrak{M}_{i}$ since $\rho \mid \mathfrak{M}_{i}$ is by assumption a pure state of $\mathfrak{M}_{i}$. Let

$$
\mathfrak{N}_{i}=E_{i} \mathfrak{M}_{i} E_{i} \oplus\left(I-E_{i}\right) \mathfrak{M}_{i}\left(I-E_{i}\right) .
$$

$\left\{\mathfrak{N}_{i}: i=1,2, \cdots\right\}$ is a strictly ascending sequence of finite dimensional von Neumann algebras in $\mathfrak{A}_{0}$. We show that $\bigcup_{i} \mathfrak{N}_{i}$ is dense in $\mathfrak{A}_{0}$. Let $A=A$ * in $\mathfrak{A}_{0}$, let $A_{i}=A_{i}^{*}$ in $\mathfrak{M}_{i}$ be such that $A_{i} \rightarrow A$. Let $z_{i}=(I-[x]) A_{i} x$. Since $x$ is a unit vector in $E_{i}$ and since $E_{i} A_{i}\left(I-E_{i}\right) A_{i} E_{i}$ is a scalar multiple of $E_{i}$, $\left\|E_{i} A_{i}\left(I-E_{i}\right) A_{i} E_{i}\right\|=\left(E_{i} A_{i}\left(I-E_{i}\right) A_{i} E_{i} x, x\right)$ and

$$
\begin{aligned}
\left\|z_{i}\right\| & \geqq\left\|\left(I-E_{i}\right) z_{i}\right\|=\left\|\left(I-E_{i}\right) A_{i} E_{i} x\right\|=\left(E_{i} A_{i}\left(I-E_{i}\right) A_{i} E_{i} x, x\right)^{1 / 2} \\
& =\left\|E_{i} A_{i}\left(I-E_{i}\right) A_{i} E_{i}\right\|^{1 / 2}=\left\|\left(I-E_{i}\right) A_{i} E_{i}\right\|=\left\|E_{i} A_{i}\left(I-E_{i}\right)\right\| .
\end{aligned}
$$

Since $z_{i} \rightarrow 0$,

$$
E_{i} A_{i} E_{i}+\left(I-E_{i}\right) A_{i}\left(I-E_{i}\right) \rightarrow A
$$

and $\bigcup_{i} \mathfrak{N}_{i}$ is dense in $\mathfrak{A}_{0}$. We have proved that $\mathfrak{A}_{0}$ satisfies (1), (2) and (3).

6. Appendix. For completeness we include the proof of certain known results about linear functionals on factors of type $I_{n}$.

Lemмa 6.1. Let $\mathfrak{M}$ be a factor of type $\mathrm{I}_{n}$, let $\left\{e_{i j}: i, j=1, \cdots, n\right\}$ be $a$ family of matrix units for $\mathfrak{M}$. Let $\phi$ be the mapping from $\mathfrak{M}^{*}$, the dual of the vector space $\mathfrak{M}$, to $\mathfrak{M}$ defined by

$$
\phi(f)=\sum_{i j} f\left(e_{j i}\right) e_{i j}
$$

for $f \in \mathfrak{M}^{*}$. Then $\phi$ is a linear and an order isomorphism, and if $\mathfrak{M}$ is identified with the algebra of all operators on $n$-dimensional Hilbert space $\mathfrak{S}$ and if $x$ is a unit vector in $\mathfrak{S}$, then $\phi\left(\omega_{x}\right)=[x]$. The mapping $\phi$ does not depend upon the choice of matrix units $\left\{e_{i j}\right\}$ in $\mathfrak{M}$. If $f \in \mathfrak{M}^{*}$ and $f \geqq 0$ then $f=\operatorname{trace}\left(\phi(f)^{1 / 2} \cdot \phi(f)^{1 / 2}\right)$

Proof. It is well known that $\phi$ is a linear isomorphism. Let $\left\{e_{1}, \cdots, e_{n}\right\}$ be an orthonormal basis for $\mathfrak{S}$ such that $e_{i j} e_{j}=e_{i}, i, j=1, \cdots, n$, let $x=\sum_{i} \gamma_{i} e_{i}$ be a unit vector in $\mathfrak{S}$. We show that $\phi\left(\omega_{x}\right)=[x]$. In fact 


$$
\left([x] e_{i}, e_{j}\right)=\left(\left(e_{i}, x\right) x, e_{j}\right)=\bar{\gamma}_{i} \gamma_{j}
$$

and

$$
\left(\phi\left(\omega_{x}\right) e_{i}, e_{j}\right)=\omega_{x}\left(e_{i j}\right)=\gamma_{j} \bar{\gamma}_{i} .
$$

By linearity we conclude that $\phi$ is an order isomorphism and that $\phi$ does not depend on the choice of the matrix units $\left\{e_{i j}\right\}$. If $f \in \mathfrak{M}^{*}$ and $f \geqq 0$ then $\phi(f)$ $\geqq 0$ and we may assume by what we have proved that $e_{i}$ is an eigenvector of $\phi(f)$, for $i=1, \cdots, n$. That is, $\phi(f)=\sum_{i} \alpha_{i} e_{i i}$ with $\alpha_{i} \geqq 0$ and hence

$$
\begin{aligned}
f & =\sum_{i} \alpha_{i} \omega_{e_{i}} \\
& =\operatorname{trace}\left(\sum_{i} \alpha_{i} e_{i i}\right) \\
& =\operatorname{trace}\left(\phi(f)^{1 / 2} \cdot \phi(f)^{1 / 2}\right) .
\end{aligned}
$$

This completes the proof of Lemma 6.1.

\section{BIBLIOGRAPHY} Math.

1. J. Glimm and R. V. Kadison, Unitary operators in $C^{*}$-algebras, to appear in Pacific J.

2. R. V. Kadison, Irreducible operator algebras, Proc. Nat. Acad. Sci. U.S.A. vol. 43 (1957) pp. 273-276.

3. I. Kaplansky, A theorem on rings of operators, Pacific J. Math. vol. 1 (1951) pp. 227-232.

4. R. Murray and J. von Neumann, On rings of operators, IV, Ann. of Math. vol. 44 (1943) pp. 716-808.

5. J. von Neumann, Approximative properties of matrices of high finite order, Portugal. Math. vol. 3 (1942) pp. 1-62.

6. I. Segal, Irreducible representations of operator algebras, Bull. Amer. Math. Soc. vol. 53 (1947) pp. 73-88.

7. H. Yoshizawa, Some remarks on unitary representations of the free group, Osaka Math. J. vol. 3 (1951) pp. 55-63.

Columbia University,

New YoRk, NEw YoRK 